\title{
Iterative algorithms with regularization for hierarchical variational inequality problems and convex minimization problems
}

\author{
Lu-Chuan Ceng ${ }^{1,2}$, Suliman Al-Homidan ${ }^{3 *}$ and Qamrul Hasan Ansari ${ }^{4}$
}

"Correspondence:

homidan@kfupm.edu.sa

${ }^{3}$ Department of Mathematics and

Statistics, King Fahd University of

Petroleum and Minerals, Dhahran,

Saudi Arabia

Full list of author information is

available at the end of the article

\begin{abstract}
In this paper, we consider a variational inequality problem which is defined over the set of intersections of the set of fixed points of a $\zeta$-strictly pseudocontractive mapping, the set of fixed points of a nonexpansive mapping and the set of solutions of a minimization problem. We propose an iterative algorithm with regularization to solve such a variational inequality problem and study the strong convergence of the sequence generated by the proposed algorithm. The results of this paper improve and extend several known results in the literature.
\end{abstract}

\section{Introduction}

Let $H$ be a real Hilbert space with the inner product $\langle\cdot, \cdot\rangle$ and the norm $\|\cdot\|$, let $C$ be a nonempty closed convex subset of $H$, and let $f: C \rightarrow \mathbf{R}$ be a convex and continuously Fréchet differentiable functional. We consider the following minimization problem (MP):

$$
\min _{x \in C} f(x)
$$

We denote by $\Xi$ the set of minimizers of problem (1.1), and we assume that $\Xi \neq \emptyset$. The gradient-projection algorithm (GPA) is one of the most elegant methods to solve the minimization problem (1.1). The convergence of the sequence generated by the GPA depends on the behavior of the gradient $\nabla f$. If $\nabla f$ is strongly monotone and Lipschitz continuous, then we get the strong convergence of the sequence generated by the GPA to a unique solution of MP (1.1). However, if the gradient $\nabla f$ is assumed to be only Lipschitz continuous, then the sequence generated by the GPA converges weakly if $H$ is infinite-dimensional (a counterexample is given in [1]). Since the Lipschitz continuity of the gradient $\nabla f$ implies that it is actually inverse strongly monotone (ism) [2], its complement can be an averaged mapping (that is, it can be expressed as a proper convex combination of the identity mapping and a nonexpansive mapping) [1]. Consequently, the GPA can be rewritten as the composite of a projection and an averaged mapping, which is again an averaged mapping. This shows that averaged mappings play an important role in the GPA. Very recently, Xu [1] used averaged mappings to study the convergence analysis of the GPA, which is an operator-oriented approach. He showed that the sequence generated by the GPA converges in norm to a minimizer of MP (1.1), which is also a unique solution of a particular type of variational inequality problem (VIP). It is worth to emphasize that the regular- 
ization, in particular the traditional Tikhonov regularization, is usually used to solve illposed optimization problems. The advantage of a regularization method is its possible strong convergence to the minimum-norm solution of the optimization problem. In [1], $\mathrm{Xu}$ introduced a hybrid gradient-projection algorithm with regularization and proved the strong convergence of the sequence to the minimum-norm solution of MP (1.1). Some iterative algorithms with or without regularization for MP (1.1) are proposed and analyzed in [3-5] for finding a common solution of MP (1.1) and the set of solutions of a nonexpansive mapping.

On the other hand, the theory of variational inequalities $[6,7]$ has emerged as an important tool to study a wide class of problems from science, engineering, social sciences. If the underlying set in the formulation of a variational inequality problem is a set of fixed points of a mapping or, more precisely, of a nonexpansive mapping, then the variational inequality problem is called hierarchical variational problem. For further details on hierarchical variational inequalities, we refer to [8-11] and the references therein.

In this paper, we consider a variational inequality problem which is defined over the set of intersections of the set of fixed points of a $\zeta$-strictly pseudocontractive mapping, the set of fixed points of a nonexpansive mapping and the set of solutions of MP (1.1). We propose an iterative algorithm with regularization to solve such a variational inequality problem and study the strong convergence of the sequence generated by the proposed algorithm. The results of this paper improve and extend several known results in the literature.

\section{Preliminaries and formulations}

Throughout the paper, unless otherwise specified, we use the following assumptions and notations. Let $H$ be a real Hilbert space whose inner product and norm are denoted by $\langle\cdot, \cdot\rangle$ and $\|\cdot\|$, respectively. Let $C$ be a nonempty closed convex subset of $H$. We write $x_{n} \rightarrow x$ (respectively, $x_{n} \rightarrow x$ ) to indicate that the sequence $\left\{x_{n}\right\}$ converges strongly (respectively, weakly) to $x$. Moreover, we use $\omega_{w}\left(x_{n}\right)$ to denote the weak $\omega$-limit set of the sequence $\left\{x_{n}\right\}$, that is,

$$
\omega_{w}\left(x_{n}\right):=\left\{x \in H: x_{n_{i}} \rightarrow x \text { for some subsequence }\left\{x_{n_{i}}\right\} \text { of }\left\{x_{n}\right\}\right\} .
$$

The metric (or nearest point) projection from $H$ onto $C$ is the mapping $P_{C}: H \rightarrow C$ which assigns to each point $x \in H$ the unique point $P_{C} x \in C$ satisfying

$$
\left\|x-P_{C} x\right\|=\inf _{y \in C}\|x-y\|=: d(x, C)
$$

Some important properties of projections are gathered in the following proposition.

Proposition 2.1 For given $x \in H$ and $z \in C$, we have

(a) $z=P_{C} x \Leftrightarrow\langle x-z, y-z\rangle \leq 0, \forall y \in C$;

(b) $z=P_{C} x \Leftrightarrow\|x-z\|^{2} \leq\|x-y\|^{2}-\|y-z\|^{2}, \forall y \in C$;

(c) $\left\langle P_{C} x-P_{C} y, x-y\right\rangle \geq\left\|P_{C} x-P_{C} y\right\|^{2}, \forall y \in H$, which concludes that $P_{C}$ is nonexpansive and monotone.

Definition 2.1 A mapping $T: H \rightarrow H$ is said to be 
(a) $\zeta$-strictly pseudocontractive if there exists a constant $\zeta \in[0,1)$ such that

$$
\|T x-T y\|^{2} \leq\|x-y\|^{2}+\zeta\|(I-T) x-(I-T) y\|^{2}, \quad \forall x, y \in H .
$$

If $\zeta=0$, then it is called nonexpansive;

(b) firmly nonexpansive if $2 T-I$ is nonexpansive, or equivalently,

$$
\langle x-y, T x-T y\rangle \geq\|T x-T y\|^{2}, \quad \forall x, y \in H ;
$$

alternatively, $T$ is firmly nonexpansive if and only if $T$ can be expressed as

$$
T=\frac{1}{2}(I+S)
$$

where $S: H \rightarrow H$ is a nonexpansive mapping.

It can be easily seen that the projection mappings are firmly nonexpansive. It is clear that $T: C \subseteq H \rightarrow C$ is $\zeta$-strictly pseudocontractive if and only if

$$
\langle T x-T y, x-y\rangle \leq\|x-y\|^{2}-\frac{1-\zeta}{2}\|(I-T) x-(I-T) y\|^{2}, \quad \forall x, y \in C .
$$

Definition 2.2 Let $T$ be a nonlinear operator with domain $D(T) \subseteq H$ and range $R(T) \subseteq H$.

(a) $T$ is said to be monotone if

$$
\langle x-y, T x-T y\rangle \geq 0, \quad \forall x, y \in D(T) .
$$

(b) Given a number $\beta>0, T$ is said to be $\beta$-strongly monotone if

$$
\langle x-y, T x-T y\rangle \geq \beta\|x-y\|^{2}, \quad \forall x, y \in D(T) .
$$

(c) Given a number $v>0, T$ is said to be $v$-inverse strongly monotone ( $v$-ism) if

$$
\langle x-y, T x-T y\rangle \geq v\|T x-T y\|^{2}, \quad \forall x, y \in D(T) .
$$

Clearly,

- if $T$ is nonexpansive, then $I-T$ is monotone;

- a projection $P_{K}$ is 1 -ism;

- if $T$ is a $\zeta$-strictly pseudocontractive mapping, then $I-T$ is $\frac{1-\zeta}{2}$-inverse strongly monotone.

Definition 2.3 [1] A mapping $T: H \rightarrow H$ is said to be an averaged mapping if it can be written as the average of the identity $I$ and a nonexpansive mapping, that is,

$$
T \equiv(1-\alpha) I+\alpha S,
$$

where $\alpha \in(0,1)$ and $S: H \rightarrow H$ is a nonexpansive mapping. More precisely, when the last equality holds, we say that $T$ is $\alpha$-averaged. Thus, firmly nonexpansive mappings (in particular, projections) are $\frac{1}{2}$-averaged maps. 
Proposition 2.2 [12] Let $T: H \rightarrow H$ be a given mapping.

(a) $T$ is nonexpansive if and only if the complement $I-T$ is $\frac{1}{2}$-ism.

(b) If $T$ is $v$-ism, then for $\gamma>0, \gamma T$ is $\frac{v}{\gamma}$-ism.

(c) $T$ is averaged if and only if the complement $I-T$ is $v$-ism for some $v>1 / 2$. Indeed, for $\alpha \in(0,1), T$ is $\alpha$-averaged if and only if $I-T$ is $\frac{1}{2 \alpha}$-ism.

Proposition 2.3 $[12,13]$ Let $S, T, V: H \rightarrow H$ be given operators.

(a) If $T=(1-\alpha) S+\alpha V$ for some $\alpha \in(0,1)$ and if $S$ is averaged and $V$ is nonexpansive, then $T$ is averaged.

(b) $T$ is firmly nonexpansive if and only if the complement $I-T$ is firmly nonexpansive.

(c) If $T=(1-\alpha) S+\alpha V$ for some $\alpha \in(0,1)$ and if $S$ is firmly nonexpansive and $V$ is nonexpansive, then $T$ is averaged.

(d) The composite of finitely many averaged mappings is averaged, that is, if each of the mappings $\left\{T_{i}\right\}_{i=1}^{N}$ is averaged, then so is the composite $T_{1} \cdots T_{N}$. In particular, if $T_{1}$ is $\alpha_{1}$-averaged and $T_{2}$ is $\alpha_{2}$-averaged, where $\alpha_{1}, \alpha_{2} \in(0,1)$, then the composite $T_{1} T_{2}$ is $\alpha$-averaged, where $\alpha=\alpha_{1}+\alpha_{2}-\alpha_{1} \alpha_{2}$.

Lemma 2.1 [14, Proposition 2.1] Let C be a nonempty closed convex subset of a real Hilbert space $H$, and let $T: C \rightarrow C$ be a mapping.

(a) If $T$ is a $\zeta$-strictly pseudocontractive mapping, then $T$ satisfies the Lipschitz condition

$$
\|T x-T y\| \leq \frac{1+\zeta}{1-\zeta}\|x-y\|, \quad \forall x, y \in C
$$

(b) If $T$ is a $\zeta$-strictly pseudocontractive mapping, then the mapping $I-T$ is semiclosed at 0 , that is, if $\left\{x_{n}\right\}$ is a sequence in $C$ such that $x_{n} \rightarrow \tilde{x}$ weakly and $(I-T) x_{n} \rightarrow 0$ strongly, then $(I-T) \tilde{x}=0$.

(c) If $T$ is a $\zeta$-(quasi-)strict pseudocontraction, then the fixed point set $\operatorname{Fix}(T)$ of $T$ is closed and convex so that the projection $P_{\mathrm{Fix}(T)}$ is well defined.

The following lemma is an immediate consequence of an inner product.

Lemma 2.2 In a real Hilbert space $H$, we have

$$
\|x+y\|^{2} \leq\|x\|^{2}+2\langle y, x+y\rangle, \quad \forall x, y \in H .
$$

The following elementary result on real sequences is quite well known.

Lemma 2.3 [15] Let $\left\{a_{n}\right\}$ be a sequence of nonnegative real numbers such that

$$
a_{n+1} \leq\left(1-s_{n}\right) a_{n}+s_{n} t_{n}+\epsilon_{n}, \quad \forall n \geq 0,
$$

where $\left\{s_{n}\right\} \subset(0,1]$ and $\left\{t_{n}\right\}$ satisfy the following conditions:

(i) $\sum_{n=0}^{\infty} s_{n}=\infty$;

(ii) either $\limsup _{n \rightarrow \infty} t_{n} \leq 0$ or $\sum_{n=0}^{\infty} s_{n}\left|t_{n}\right|<\infty$;

(iii) $\sum_{n=0}^{\infty} \epsilon_{n}<\infty$, where $\epsilon_{n} \geq 0, \forall n \geq 0$.

Then $\lim _{n \rightarrow \infty} a_{n}=0$. 
Lemma 2.4 [10] Let $C$ be a nonempty closed convex subset of a real Hilbert space $H$, and let $T: C \rightarrow C$ be a $\zeta$-strictly pseudocontractive mapping. Let $\gamma$ and $\delta$ be two nonnegative real numbers such that $(\gamma+\delta) \zeta \leq \gamma$. Then

$$
\|\gamma(x-y)+\delta(T x-T y)\| \leq(\gamma+\delta)\|x-y\|, \quad \forall x, y \in C .
$$

The following lemma appeared implicitly in the paper of Reineermann [16].

Lemma 2.5 [16] Let $H$ be a real Hilbert space. Then, for all $x, y \in H$ and $\lambda \in[0,1]$,

$$
\|\lambda x+(1-\lambda) y\|^{2}=\lambda\|x\|^{2}+(1-\lambda)\|y\|^{2}-\lambda(1-\lambda)\|x-y\|^{2} .
$$

Let $C$ be a nonempty closed convex subset of a real Hilbert space $H$, and let $A: C \rightarrow H$ be a monotone mapping. The variational inequality problem (VIP) is to find $x \in C$ such that

$$
\langle A x, y-x\rangle \geq 0, \quad \forall y \in C
$$

The solution set of the VIP is denoted by $\operatorname{VI}(C, A)$. It is well known that

$$
x \in \operatorname{VI}(C, A) \quad \Leftrightarrow \quad x=P_{C}(x-\lambda A x), \quad \forall \lambda>0 .
$$

A set-valued mapping $V: H \rightarrow 2^{H}$ is called monotone if for all $x, y \in H, f \in V x$ and $g \in V y$ imply that $\langle x-y, f-g\rangle \geq 0$. A monotone set-valued mapping $V: H \rightarrow 2^{H}$ is called maximal if its graph $\operatorname{Gph}(V)$ is not properly contained in the graph of any other monotone setvalued mapping. It is known that a monotone set-valued mapping $V: H \rightarrow 2^{H}$ is maximal if and only if for $(x, f) \in H \times H,\langle x-y, f-g\rangle \geq 0$ for every $(y, g) \in \mathrm{Gph}(V)$ implies that $f \in V x$. Let $A: C \rightarrow H$ be a monotone and Lipschitz continuous mapping and $N_{C} v$ be the normal cone to $C$ at $v \in C$, that is,

$$
N_{C} v=\{w \in H:\langle v-u, w\rangle \geq 0, \forall u \in C\} .
$$

Define

$$
V v= \begin{cases}A v+N_{C} v & \text { if } v \in C, \\ \varnothing & \text { if } v \notin C .\end{cases}
$$

Lemma 2.6 [17] Let $A: C \rightarrow H$ be a monotone mapping. Then

(i) $V$ is maximal monotone;

(ii) $v \in V^{-1} 0 \Leftrightarrow v \in \mathrm{VI}(C, A)$.

Throughout the paper, we denote by $\operatorname{Fix}(T)$ and $\operatorname{Fix}(\Gamma)$ the set of fixed points of $T$ and $\Gamma$, respectively. We also assume that the set $\operatorname{Fix}(T) \cap \operatorname{Fix}(\Gamma) \cap \Xi$ is nonempty closed and convex.

Let $S, T: C \rightarrow C$ be nonexpansive mappings and $\Gamma: C \rightarrow C$ be a $\zeta$-strictly pseudocontractive mapping with $\zeta \in[0,1)$. In this paper, we consider and study the following 
hierarchical variational inequality problem which is defined on $\operatorname{Fix}(T) \cap \operatorname{Fix}(\Gamma) \cap \Xi$.

Find $\tilde{x} \in \operatorname{Fix}(T) \cap \operatorname{Fix}(\Gamma) \cap \Xi$ such that

$$
\langle\tilde{x}-S \tilde{x}, \tilde{x}-x\rangle \leq 0, \quad \forall x \in \operatorname{Fix}(T) \cap \operatorname{Fix}(\Gamma) \cap \Xi .
$$

We denote by $\Omega$ the solution set of problem (2.1). It is not difficult to verify that solving (2.1) is equivalent to the fixed point problem of finding $\tilde{x} \in C$ such that

$$
\tilde{x}=P_{\operatorname{Fix}(T) \cap \operatorname{Fix}(\Gamma) \cap \Xi S \tilde{x},}
$$

where $P_{\operatorname{Fix}(T) \cap \mathrm{Fix}(\Gamma) \cap \Xi}$ stands for the metric projection onto the closed convex set $\operatorname{Fix}(T) \cap$ $\operatorname{Fix}(\Gamma) \cap \Xi$.

Problem (2.1) contains the hierarchical variational inequality problems considered and studied in $[8,18,19]$ and the references therein.

By using the definition of the normal cone to $\operatorname{Fix}(T) \cap \operatorname{Fix}(\Gamma) \cap \Xi$, we have the mapping $N_{\mathrm{Fix}(T) \cap \mathrm{Fix}(\Gamma) \cap \Xi}: H \rightarrow 2^{H}:$

$$
x \mapsto\left\{\begin{array}{l}
\{u \in H:(\forall y \in \operatorname{Fix}(T) \cap \operatorname{Fix}(\Gamma) \cap \Xi)\langle y-x, u\rangle \leq 0\}, \\
\quad \text { if } x \in \operatorname{Fix}(T) \cap \operatorname{Fix}(\Gamma) \cap \Xi ; \\
\emptyset, \quad \text { otherwise }
\end{array}\right.
$$

and we readily prove that (2.1) is equivalent to the variational inequality

$$
0 \in(I-S) \tilde{x}+N_{\operatorname{Fix}(T) \cap \operatorname{Fix}(\Gamma) \cap \Xi \tilde{x} .}
$$

By combining the hybrid gradient-projection method of $\mathrm{Xu}$ [1] and a two-step method of Yao et al. [11], we introduce the following three-step iterative algorithm:

$$
\left\{\begin{array}{l}
y_{n}=\theta_{n} S x_{n}+\left(1-\theta_{n}\right) x_{n}, \\
z_{n}=\beta_{n} Q y_{n}+\left(1-\beta_{n}\right) T P_{C}\left(y_{n}-\lambda \nabla f_{\alpha_{n}}\left(y_{n}\right)\right), \\
x_{n+1}=\sigma_{n} z_{n}+\gamma_{n} P_{C}\left(z_{n}-\lambda \nabla f_{\alpha_{n}}\left(z_{n}\right)\right)+\delta_{n} \Gamma P_{C}\left(z_{n}-\lambda \nabla f_{\alpha_{n}}\left(z_{n}\right)\right), \quad \forall n \geq 0,
\end{array}\right.
$$

where $Q: C \rightarrow C$ is a $\rho$-contraction mapping, $\left\{\alpha_{n}\right\} \subset(0, \infty),\left\{\beta_{n}\right\},\left\{\theta_{n}\right\},\left\{\sigma_{n}\right\} \subset(0,1)$ and $\left\{\gamma_{n}\right\},\left\{\delta_{n}\right\} \subset[0,1]$ with $\sigma_{n}+\gamma_{n}+\delta_{n}=1, \forall n \geq 0$. It is proven that under appropriate assumptions, the above iterative sequence $\left\{x_{n}\right\}$ converges strongly to an element $\tilde{x} \in \operatorname{Fix}(T) \cap$ $\operatorname{Fix}(\Gamma) \cap \Xi$.

\section{Main results}

Let us consider the following assumptions:

- the mapping $Q: C \rightarrow C$ is a $\rho$-contraction;

- the mapping $\Gamma: C \rightarrow C$ is a $\zeta$-strict pseudocontraction;

- $S, T: C \rightarrow C$ are two nonexpansive mappings;

- $\nabla f: C \rightarrow H$ is Lipschitz continuous with $0<\lambda<\frac{2}{L}$;

- $\left\{\alpha_{n}\right\}$ is a sequence in $(0, \infty)$ with $\sum_{n=0}^{\infty} \alpha_{n}<\infty$;

- $\left\{\beta_{n}\right\},\left\{\theta_{n}\right\},\left\{\sigma_{n}\right\}$ are sequences in $(0,1)$ with $0<\liminf _{n \rightarrow \infty} \sigma_{n} \leq \limsup _{n \rightarrow \infty} \sigma_{n}<1$; 
- $\left\{\gamma_{n}\right\},\left\{\delta_{n}\right\}$ are sequences in $[0,1]$ with $\sigma_{n}+\gamma_{n}+\delta_{n}=1, \forall n \geq 0$;

- $\liminf _{n \rightarrow \infty} \delta_{n}>0$ and $\left(\gamma_{n}+\delta_{n}\right) \zeta \leq \gamma_{n}, \forall n \geq 0$.

Theorem 3.1 Let $\left\{x_{n}\right\}$ be a bounded sequence generated from any given $x_{0} \in C$ by (2.2). Assume that the following conditions hold:

(H1) $\sum_{n=0}^{\infty} \beta_{n}=\infty, \lim _{n \rightarrow \infty} \frac{1}{\beta_{n}}\left|1-\frac{\theta_{n-1}}{\theta_{n}}\right|=0$;

(H2) $\lim _{n \rightarrow \infty} \frac{1}{\beta_{n}}\left|\frac{1}{\theta_{n}}-\frac{1}{\theta_{n-1}}\right|=0, \lim _{n \rightarrow \infty} \frac{1}{\theta_{n}}\left|1-\frac{\beta_{n-1}}{\beta_{n}}\right|=0$;

(H3) $\lim _{n \rightarrow \infty} \theta_{n}=0$ and $\lim _{n \rightarrow \infty} \frac{\alpha_{n}+\beta_{n}}{\theta_{n}}=0$;

(H4) $\lim _{n \rightarrow \infty} \frac{\left|\alpha_{n}-\alpha_{n-1}\right|}{\beta_{n} \theta_{n}}=0, \lim _{n \rightarrow \infty} \frac{\left|\sigma_{n}-\sigma_{n-1}\right|}{\beta_{n} \theta_{n}}=0$;

(H5) $\lim _{n \rightarrow \infty} \frac{1}{\beta_{n} \theta_{n}}\left|\frac{\gamma_{n}}{1-\sigma_{n}}-\frac{\gamma_{n-1}}{1-\sigma_{n-1}}\right|=0$.

Then the following assertions hold:

(i) $\lim _{n \rightarrow \infty} \frac{\left\|x_{n+1}-x_{n}\right\|}{\theta_{n}}=0$;

(ii) $\omega_{w}\left(x_{n}\right) \subset \Omega$.

Proof First of all, we show that $P_{C}\left(I-\lambda \nabla f_{\alpha}\right)$ is $\xi$-averaged for each $\lambda \in\left(0, \frac{2}{\alpha+L}\right)$, where

$$
\xi=\frac{2+\lambda(\alpha+L)}{4} \in(0,1) .
$$

Indeed, the Lipschitz continuity of $\nabla f$ implies that $\nabla f$ is $\frac{1}{L}$-ism [2], that is,

$$
\langle\nabla f(x)-\nabla f(y), x-y\rangle \geq \frac{1}{L}\|\nabla f(x)-\nabla f(y)\|^{2} .
$$

Observe that

$$
\begin{aligned}
(\alpha & +L)\left\langle\nabla f_{\alpha}(x)-\nabla f_{\alpha}(y), x-y\right\rangle \\
& =(\alpha+L)\left[\alpha\|x-y\|^{2}+\langle\nabla f(x)-\nabla f(y), x-y\rangle\right] \\
& =\alpha^{2}\|x-y\|^{2}+\alpha\langle\nabla f(x)-\nabla f(y), x-y\rangle+\alpha L\|x-y\|^{2}+L\langle\nabla f(x)-\nabla f(y), x-y\rangle \\
& \geq \alpha^{2}\|x-y\|^{2}+2 \alpha\langle\nabla f(x)-\nabla f(y), x-y\rangle+\|\nabla f(x)-\nabla f(y)\|^{2} \\
& =\|\alpha(x-y)+\nabla f(x)-\nabla f(y)\|^{2} \\
& =\left\|\nabla f_{\alpha}(x)-\nabla f_{\alpha}(y)\right\|^{2} .
\end{aligned}
$$

Therefore, it follows that $\nabla f_{\alpha}=\alpha I+\nabla f$ is $\frac{1}{\alpha+L}$-ism. Thus, by Proposition 2.2(b), $\lambda \nabla f_{\alpha}$ is $\frac{1}{\lambda(\alpha+L)}$-ism. From Proposition 2.2(c), the complement $I-\lambda \nabla f_{\alpha}$ is $\frac{\lambda(\alpha+L)}{2}$-averaged. Therefore, noting that $P_{C}$ is $\frac{1}{2}$-averaged and utilizing Proposition $2.3(\mathrm{~d})$, we obtain that for each $\lambda \in\left(0, \frac{2}{\alpha+L}\right), P_{C}\left(I-\lambda \nabla f_{\alpha}\right)$ is $\xi$-averaged with

$$
\xi=\frac{1}{2}+\frac{\lambda(\alpha+L)}{2}-\frac{1}{2} \cdot \frac{\lambda(\alpha+L)}{2}=\frac{2+\lambda(\alpha+L)}{4} \in(0,1) .
$$

This shows that $P_{C}\left(I-\lambda \nabla f_{\alpha}\right)$ is nonexpansive. For $\lambda \in\left(0, \frac{2}{L}\right)$, utilizing the fact that $\lim _{n \rightarrow \infty} \frac{2}{\alpha_{n}+L}=\frac{2}{L}$, we may assume that

$$
0<\lambda<\frac{2}{\alpha_{n}+L}, \quad \forall n \geq 0 .
$$


Consequently, it follows that for each integer $n \geq 0, P_{C}\left(I-\lambda \nabla f_{\alpha_{n}}\right)$ is $\xi_{n}$-averaged with

$$
\xi_{n}=\frac{1}{2}+\frac{\lambda\left(\alpha_{n}+L\right)}{2}-\frac{1}{2} \cdot \frac{\lambda\left(\alpha_{n}+L\right)}{2}=\frac{2+\lambda\left(\alpha_{n}+L\right)}{4} \in(0,1)
$$

This implies that $P_{C}\left(I-\lambda \nabla f_{\alpha_{n}}\right)$ is nonexpansive for all $n \geq 0$.

The rest of the proof is divided into several steps.

STEP 1. $\lim _{n \rightarrow \infty} \frac{\left\|x_{n+1}-x_{n}\right\|}{\theta_{n}}=0$.

For simplicity, we put $\tilde{y}_{n}=P_{C}\left(y_{n}-\lambda \nabla f_{\alpha_{n}}\left(y_{n}\right)\right)$ and $\tilde{z}_{n}=P_{C}\left(z_{n}-\lambda \nabla f_{\alpha_{n}}\left(z_{n}\right)\right)$ for every $n \geq 0$.

Then $z_{n}=\beta_{n} Q y_{n}+\left(1-\beta_{n}\right) T \tilde{y}_{n}$ and $x_{n+1}=\sigma_{n} z_{n}+\gamma_{n} \tilde{z}_{n}+\delta_{n} \Gamma \tilde{z}_{n}$ for every $n \geq 0$.

Taking into account $0<\liminf _{n \rightarrow \infty} \sigma_{n} \leq \limsup _{n \rightarrow \infty} \sigma_{n}<1$, without loss of generality, we may assume that $\left\{\sigma_{n}\right\} \subset[c, d]$ for some $c, d \in(0,1)$. We write $x_{n}=\sigma_{n-1} z_{n-1}+\left(1-\sigma_{n-1}\right) v_{n-1}$, $\forall n \geq 1$, where $v_{n-1}=\frac{x_{n}-\sigma_{n-1} z_{n-1}}{1-\sigma_{n-1}}$. It follows that for all $n \geq 1$,

$$
\begin{aligned}
v_{n}-v_{n-1}= & \frac{x_{n+1}-\sigma_{n} z_{n}}{1-\sigma_{n}}-\frac{x_{n}-\sigma_{n-1} z_{n-1}}{1-\sigma_{n-1}} \\
= & \frac{\gamma_{n} \tilde{z}_{n}+\delta_{n} \Gamma \tilde{z}_{n}}{1-\sigma_{n}}-\frac{\gamma_{n-1} \tilde{z}_{n-1}+\delta_{n-1} \Gamma \tilde{z}_{n-1}}{1-\sigma_{n-1}} \\
= & \frac{\gamma_{n}\left(\tilde{z}_{n}-\tilde{z}_{n-1}\right)+\delta_{n}\left(\Gamma \tilde{z}_{n}-\Gamma \tilde{z}_{n-1}\right)}{1-\sigma_{n}} \\
& +\left(\frac{\gamma_{n}}{1-\sigma_{n}}-\frac{\gamma_{n-1}}{1-\sigma_{n-1}}\right) \tilde{z}_{n-1}+\left(\frac{\delta_{n}}{1-\sigma_{n}}-\frac{\delta_{n-1}}{1-\sigma_{n-1}}\right) \Gamma \tilde{z}_{n-1} .
\end{aligned}
$$

Since $\left(\gamma_{n}+\delta_{n}\right) \zeta \leq \gamma_{n}$ for all $n \geq 0$, by Lemma 2.4, we have

$$
\left\|\gamma_{n}\left(\tilde{z}_{n}-\tilde{z}_{n-1}\right)+\delta_{n}\left(\Gamma \tilde{z}_{n}-\Gamma \tilde{z}_{n-1}\right)\right\| \leq\left(\gamma_{n}+\delta_{n}\right)\left\|\tilde{z}_{n}-\tilde{z}_{n-1}\right\| .
$$

Now, we estimate $\left\|z_{n}-z_{n-1}\right\|$. Observe that for every $n \geq 1$,

$$
\begin{aligned}
\left\|\tilde{y}_{n}-\tilde{y}_{n-1}\right\| \leq & \left\|P_{C}\left(I-\lambda \nabla f_{\alpha_{n}}\right) y_{n}-P_{C}\left(I-\lambda \nabla f_{\alpha_{n}}\right) y_{n-1}\right\| \\
& +\left\|P_{C}\left(I-\lambda \nabla f_{\alpha_{n}}\right) y_{n-1}-P_{C}\left(I-\lambda \nabla f_{\alpha_{n-1}}\right) y_{n-1}\right\| \\
\leq & \left\|y_{n}-y_{n-1}\right\|+\left\|P_{C}\left(I-\lambda \nabla f_{\alpha_{n}}\right) y_{n-1}-P_{C}\left(I-\lambda \nabla f_{\alpha_{n-1}}\right) y_{n-1}\right\| \\
\leq & \left\|y_{n}-y_{n-1}\right\|+\left\|\left(I-\lambda \nabla f_{\alpha_{n}}\right) y_{n-1}-\left(I-\lambda \nabla f_{\alpha_{n-1}}\right) y_{n-1}\right\| \\
= & \left\|y_{n}-y_{n-1}\right\|+\left\|\lambda \nabla f_{\alpha_{n}}\left(y_{n-1}\right)-\lambda \nabla f_{\alpha_{n-1}}\left(y_{n-1}\right)\right\| \\
= & \left\|y_{n}-y_{n-1}\right\|+\lambda\left|\alpha_{n}-\alpha_{n-1}\right|\left\|y_{n-1}\right\| .
\end{aligned}
$$

Similarly, for all $n \geq 1$, we have

$$
\left\|\tilde{z}_{n}-\tilde{z}_{n-1}\right\| \leq\left\|z_{n}-z_{n-1}\right\|+\lambda\left|\alpha_{n}-\alpha_{n-1}\right|\left\|z_{n-1}\right\|
$$

From (2.2), we have

$$
\left\{\begin{array}{l}
y_{n}=\theta_{n} S x_{n}+\left(1-\theta_{n}\right) x_{n}, \\
y_{n-1}=\theta_{n-1} S x_{n-1}+\left(1-\theta_{n-1}\right) x_{n-1}, \quad \forall n \geq 1,
\end{array}\right.
$$


and therefore

$$
y_{n}-y_{n-1}=\theta_{n}\left(S x_{n}-S x_{n-1}\right)+\left(\theta_{n}-\theta_{n-1}\right)\left(S x_{n-1}-x_{n-1}\right)+\left(1-\theta_{n}\right)\left(x_{n}-x_{n-1}\right),
$$

which implies that

$$
\begin{aligned}
\left\|y_{n}-y_{n-1}\right\| & \leq \theta_{n}\left\|S x_{n}-S x_{n-1}\right\|+\left|\theta_{n}-\theta_{n-1}\right|\left\|S x_{n-1}-x_{n-1}\right\|+\left(1-\theta_{n}\right)\left\|x_{n}-x_{n-1}\right\| \\
& \leq\left\|x_{n}-x_{n-1}\right\|+\left|\theta_{n}-\theta_{n-1}\right|\left\|S x_{n-1}-x_{n-1}\right\| .
\end{aligned}
$$

Also, from (2.2) we have

$$
\left\{\begin{array}{l}
z_{n}=\beta_{n} Q y_{n}+\left(1-\beta_{n}\right) T \tilde{y}_{n}, \\
z_{n-1}=\beta_{n-1} Q y_{n-1}+\left(1-\beta_{n-1}\right) T \tilde{y}_{n-1}, \quad \forall n \geq 1,
\end{array}\right.
$$

then simple calculations show that

$$
z_{n}-z_{n-1}=\left(1-\beta_{n}\right)\left(T \tilde{y}_{n}-T \tilde{y}_{n-1}\right)+\left(\beta_{n}-\beta_{n-1}\right)\left(Q y_{n-1}-T \tilde{y}_{n-1}\right)+\beta_{n}\left(Q y_{n}-Q y_{n-1}\right),
$$

and thus, from (3.3)-(3.4), we have

$$
\begin{aligned}
\| z_{n}- & z_{n-1} \| \\
\leq & \left(1-\beta_{n}\right)\left\|T \tilde{y}_{n}-T \tilde{y}_{n-1}\right\|+\left|\beta_{n}-\beta_{n-1}\right|\left\|Q y_{n-1}-T \tilde{y}_{n-1}\right\|+\beta_{n}\left\|Q y_{n}-Q y_{n-1}\right\| \\
\leq & \left(1-\beta_{n}\right)\left\|\tilde{y}_{n}-\tilde{y}_{n-1}\right\|+\left|\beta_{n}-\beta_{n-1}\right|\left\|Q y_{n-1}-T \tilde{y}_{n-1}\right\|+\beta_{n}\left\|Q y_{n}-Q y_{n-1}\right\| \\
\leq & \left(1-\beta_{n}\right)\left(\left\|y_{n}-y_{n-1}\right\|+\lambda\left|\alpha_{n}-\alpha_{n-1}\right|\left\|y_{n-1}\right\|\right)+\left|\beta_{n}-\beta_{n-1}\right|\left\|Q y_{n-1}-T \tilde{y}_{n-1}\right\| \\
& +\beta_{n} \rho\left\|y_{n}-y_{n-1}\right\| \\
\leq & \left(1-(1-\rho) \beta_{n}\right)\left\|y_{n}-y_{n-1}\right\|+\lambda\left|\alpha_{n}-\alpha_{n-1}\right|\left\|y_{n-1}\right\|+\left|\beta_{n}-\beta_{n-1}\right|\left\|Q y_{n-1}-T \tilde{y}_{n-1}\right\| \\
\leq & \left(1-(1-\rho) \beta_{n}\right)\left[\left\|x_{n}-x_{n-1}\right\|+\left|\theta_{n}-\theta_{n-1}\right|\left\|S x_{n-1}-x_{n-1}\right\|\right] \\
& +\lambda\left|\alpha_{n}-\alpha_{n-1}\right|\left\|y_{n-1}\right\|+\left|\beta_{n}-\beta_{n-1}\right|\left\|Q y_{n-1}-T \tilde{y}_{n-1}\right\| \\
\leq & \left(1-(1-\rho) \beta_{n}\right)\left\|x_{n}-x_{n-1}\right\|+\left|\theta_{n}-\theta_{n-1}\right|\left\|S x_{n-1}-x_{n-1}\right\| \\
& +\lambda\left|\alpha_{n}-\alpha_{n-1}\right|\left\|y_{n-1}\right\|+\left|\beta_{n}-\beta_{n-1}\right|\left\|Q y_{n-1}-T \tilde{y}_{n-1}\right\| \\
\leq & \left(1-(1-\rho) \beta_{n}\right)\left\|x_{n}-x_{n-1}\right\|+M_{1}\left[\left|\theta_{n}-\theta_{n-1}\right|+\left|\alpha_{n}-\alpha_{n-1}\right|+\left|\beta_{n}-\beta_{n-1}\right|\right]
\end{aligned}
$$

where $\left\|S x_{n}-x_{n}\right\|+\lambda\left\|y_{n}\right\|+\left\|Q y_{n}-T \tilde{y}_{n}\right\| \leq M_{1}, \forall n \geq 0$ for some $M_{1}>0$. This together with (3.1)-(3.3) implies that

$$
\begin{aligned}
\| v_{n}- & v_{n-1} \| \\
\leq & \frac{\left\|\gamma_{n}\left(\tilde{z}_{n}-\tilde{z}_{n-1}\right)+\delta_{n}\left(\Gamma \tilde{z}_{n}-\Gamma \tilde{z}_{n-1}\right)\right\|}{1-\sigma_{n}}+\left|\frac{\gamma_{n}}{1-\sigma_{n}}-\frac{\gamma_{n-1}}{1-\sigma_{n-1}}\right|\left\|\tilde{z}_{n-1}\right\| \\
& +\left|\frac{\delta_{n}}{1-\sigma_{n}}-\frac{\delta_{n-1}}{1-\sigma_{n-1}}\right|\left\|\Gamma \tilde{z}_{n-1}\right\| \\
\leq & \frac{\left(\gamma_{n}+\delta_{n}\right)\left\|\tilde{z}_{n}-\tilde{z}_{n-1}\right\|}{1-\sigma_{n}}+\left|\frac{\gamma_{n}}{1-\sigma_{n}}-\frac{\gamma_{n-1}}{1-\sigma_{n-1}}\right|\left\|\tilde{z}_{n-1}\right\|+\left|\frac{\gamma_{n}}{1-\sigma_{n}}-\frac{\gamma_{n-1}}{1-\sigma_{n-1}}\right|\left\|\Gamma \tilde{z}_{n-1}\right\|
\end{aligned}
$$




$$
\begin{aligned}
= & \left\|\tilde{z}_{n}-\tilde{z}_{n-1}\right\|+\left|\frac{\gamma_{n}}{1-\sigma_{n}}-\frac{\gamma_{n-1}}{1-\sigma_{n-1}}\right|\left(\left\|\tilde{z}_{n-1}\right\|+\left\|\Gamma \tilde{y}_{n-1}\right\|\right) \\
\leq & \left\|z_{n}-z_{n-1}\right\|+\lambda\left|\alpha_{n}-\alpha_{n-1}\right|\left\|z_{n-1}\right\|+\left|\frac{\gamma_{n}}{1-\sigma_{n}}-\frac{\gamma_{n-1}}{1-\sigma_{n-1}}\right|\left(\left\|\tilde{z}_{n-1}\right\|+\left\|\Gamma \tilde{z}_{n-1}\right\|\right) \\
\leq & \left(1-(1-\rho) \beta_{n}\right)\left\|x_{n}-x_{n-1}\right\|+M_{1}\left[\left|\theta_{n}-\theta_{n-1}\right|+\left|\alpha_{n}-\alpha_{n-1}\right|+\left|\beta_{n}-\beta_{n-1}\right|\right] \\
& +\lambda\left|\alpha_{n}-\alpha_{n-1}\right|\left\|z_{n-1}\right\|+\left|\frac{\gamma_{n}}{1-\sigma_{n}}-\frac{\gamma_{n-1}}{1-\sigma_{n-1}}\right|\left(\left\|\tilde{z}_{n-1}\right\|+\left\|\Gamma \tilde{z}_{n-1}\right\|\right) \\
\leq & \left(1-(1-\rho) \beta_{n}\right)\left\|x_{n}-x_{n-1}\right\|+M_{2}\left[\left|\theta_{n}-\theta_{n-1}\right|+2\left|\alpha_{n}-\alpha_{n-1}\right|+\left|\beta_{n}-\beta_{n-1}\right|\right. \\
& \left.+\left|\frac{\gamma_{n}}{1-\sigma_{n}}-\frac{\gamma_{n-1}}{1-\sigma_{n-1}}\right|\right]
\end{aligned}
$$

where $M_{1}+\lambda\left\|z_{n}\right\|+\left\|\tilde{z}_{n}\right\|+\left\|\Gamma \tilde{z}_{n}\right\| \leq M_{2}, \forall n \geq 0$ for some $M_{2}>0$.

Further, we observe that

$$
\left\{\begin{array}{l}
x_{n+1}=\sigma_{n} z_{n}+\left(1-\sigma_{n}\right) v_{n} \\
x_{n}=\sigma_{n-1} z_{n-1}+\left(1-\beta_{n-1}\right) v_{n-1}, \quad \forall n \geq 1
\end{array}\right.
$$

and then by simple calculations, we have

$$
x_{n+1}-x_{n}=\left(1-\sigma_{n}\right)\left(v_{n}-v_{n-1}\right)+\left(\sigma_{n}-\sigma_{n-1}\right)\left(z_{n-1}-v_{n-1}\right)+\sigma_{n}\left(z_{n}-z_{n-1}\right) .
$$

By taking norm and using (3.5)-(3.6), we get

$$
\begin{aligned}
\| x_{n+1} & -x_{n} \| \\
\leq & \left(1-\sigma_{n}\right)\left\|v_{n}-v_{n-1}\right\|+\left|\sigma_{n}-\sigma_{n-1}\right|\left\|z_{n-1}-v_{n-1}\right\|+\sigma_{n}\left\|z_{n}-z_{n-1}\right\| \\
\leq & \left(1-\sigma_{n}\right)\left\{\left(1-(1-\rho) \beta_{n}\right)\left\|x_{n}-x_{n-1}\right\|+M_{2}\left[\left|\theta_{n}-\theta_{n-1}\right|+2\left|\alpha_{n}-\alpha_{n-1}\right|+\left|\beta_{n}-\beta_{n-1}\right|\right.\right. \\
& \left.\left.+\left|\frac{\gamma_{n}}{1-\sigma_{n}}-\frac{\gamma_{n-1}}{1-\sigma_{n-1}}\right|\right]\right\}+\left|\sigma_{n}-\sigma_{n-1}\right||| z_{n-1}-v_{n-1} \| \\
& +\sigma_{n}\left\{\left(1-(1-\rho) \beta_{n}\right)\left\|x_{n}-x_{n-1}\right\|+M_{1}\left[\left|\theta_{n}-\theta_{n-1}\right|+\left|\alpha_{n}-\alpha_{n-1}\right|+\left|\beta_{n}-\beta_{n-1}\right|\right]\right\} \\
\leq & \left(1-(1-\rho) \beta_{n}\right)\left\|x_{n}-x_{n-1}\right\|+M_{2}\left[\left|\theta_{n}-\theta_{n-1}\right|+2\left|\alpha_{n}-\alpha_{n-1}\right|+\left|\beta_{n}-\beta_{n-1}\right|\right. \\
& \left.+\left|\frac{\gamma_{n}}{1-\sigma_{n}}-\frac{\gamma_{n-1}}{1-\sigma_{n-1}}\right|\right]+\left|\sigma_{n}-\sigma_{n-1}\right||| z_{n-1}-v_{n-1} \| \\
\leq & \left(1-(1-\rho) \beta_{n}\right)\left\|x_{n}-x_{n-1}\right\|+M_{3}\left[\left|\theta_{n}-\theta_{n-1}\right|+2\left|\alpha_{n}-\alpha_{n-1}\right|+\left|\beta_{n}-\beta_{n-1}\right|\right. \\
& \left.+\left|\frac{\gamma_{n}}{1-\sigma_{n}}-\frac{\gamma_{n-1}}{1-\sigma_{n-1}}\right|+\left|\sigma_{n}-\sigma_{n-1}\right|\right]
\end{aligned}
$$

where $M_{2}+\left\|z_{n}-v_{n}\right\| \leq M_{3}, \forall n \geq 0$ for some $M_{3} \geq 0$. Therefore,

$$
\begin{aligned}
& \frac{\left\|x_{n+1}-x_{n}\right\|}{\theta_{n}} \\
& \quad \leq\left(1-(1-\rho) \beta_{n}\right) \frac{\left\|x_{n}-x_{n-1}\right\|}{\theta_{n}}+M_{3}\left[\frac{\left|\theta_{n}-\theta_{n-1}\right|}{\theta_{n}}+2 \frac{\left|\alpha_{n}-\alpha_{n-1}\right|}{\theta_{n}}+\frac{\left|\beta_{n}-\beta_{n-1}\right|}{\theta_{n}}\right.
\end{aligned}
$$




$$
\begin{aligned}
& \left.+\frac{1}{\theta_{n}}\left|\frac{\gamma_{n}}{1-\sigma_{n}}-\frac{\gamma_{n-1}}{1-\sigma_{n-1}}\right|+\frac{\left|\sigma_{n}-\sigma_{n-1}\right|}{\theta_{n}}\right] \\
= & \left(1-(1-\rho) \beta_{n}\right) \frac{|| x_{n}-x_{n-1} \|}{\theta_{n-1}}+\left(1-(1-\rho) \beta_{n}\right)\left(\frac{\left\|x_{n}-x_{n-1}\right\|}{\theta_{n}}-\frac{\left\|x_{n}-x_{n-1}\right\|}{\theta_{n-1}}\right) \\
& +M_{3}\left[\frac{\left|\theta_{n}-\theta_{n-1}\right|}{\theta_{n}}+2 \frac{\left|\alpha_{n}-\alpha_{n-1}\right|}{\theta_{n}}+\frac{\left|\beta_{n}-\beta_{n-1}\right|}{\theta_{n}}\right. \\
& \left.+\frac{1}{\theta_{n}}\left|\frac{\gamma_{n}}{1-\sigma_{n}}-\frac{\gamma_{n-1}}{1-\sigma_{n-1} \mid}\right| \frac{\left|\sigma_{n}-\sigma_{n-1}\right|}{\theta_{n}}\right] \\
\leq & \left(1-(1-\rho) \beta_{n}\right) \frac{|| x_{n}-x_{n-1} \mid}{\theta_{n-1}}+M\left[\left|\frac{1}{\theta_{n}}-\frac{1}{\theta_{n-1}}\right|\right. \\
& \left.+\frac{\left|\theta_{n}-\theta_{n-1}\right|}{\theta_{n}}+2 \frac{\left|\alpha_{n}-\alpha_{n-1}\right|}{\theta_{n}}+\frac{\left|\beta_{n}-\beta_{n-1}\right|}{\theta_{n}}+\frac{1}{\theta_{n}}\left|\frac{\gamma_{n}}{1-\sigma_{n}}-\frac{\gamma_{n-1}}{1-\sigma_{n-1}}\right|+\frac{\left|\sigma_{n}-\sigma_{n-1}\right|}{\theta_{n}}\right] \\
= & \left(1-(1-\rho) \beta_{n}\right) \frac{|| x_{n}-x_{n-1} \|}{\theta_{n-1}}+(1-\rho) \beta_{n} \cdot \frac{M}{1-\rho}\left\{\frac{1}{\beta_{n}}\left|\frac{1}{\theta_{n}}-\frac{1}{\theta_{n-1}}\right|\right. \\
& +\frac{1}{\beta_{n}}\left|1-\frac{\theta_{n-1}}{\theta_{n}}\right|+2 \frac{\left|\alpha_{n}-\alpha_{n-1}\right|}{\beta_{n} \theta_{n}}+\frac{1}{\theta_{n}}\left|1-\frac{\beta_{n-1}}{\beta_{n}}\right| \\
& \left.+\frac{1}{\beta_{n} \theta_{n}}\left|\frac{\gamma_{n}}{1-\sigma_{n}}-\frac{\gamma_{n-1}}{1-\sigma_{n-1}}\right|+\frac{\left|\sigma_{n}-\sigma_{n-1}\right|}{\beta_{n} \theta_{n}}\right\},
\end{aligned}
$$

where $M_{3}+\left\|x_{n}-x_{n-1}\right\| \leq M, \forall n \geq 1$ for some $M \geq 0$. From (H1)-(H5), it follows that $\sum_{n=0}^{\infty}(1-\rho) \beta_{n}=\infty$ and

$$
\begin{aligned}
& \lim _{n \rightarrow \infty} \frac{M}{1-\rho}\left\{\frac{1}{\beta_{n}}\left|\frac{1}{\theta_{n}}-\frac{1}{\theta_{n-1}}\right|+\frac{1}{\beta_{n}}\left|1-\frac{\theta_{n-1}}{\theta_{n}}\right|+2 \frac{\left|\alpha_{n}-\alpha_{n-1}\right|}{\beta_{n} \theta_{n}}\right. \\
& \left.\quad+\frac{1}{\theta_{n}}\left|1-\frac{\beta_{n-1}}{\beta_{n}}\right|+\frac{1}{\beta_{n} \theta_{n}}\left|\frac{\gamma_{n}}{1-\sigma_{n}}-\frac{\gamma_{n-1}}{1-\sigma_{n-1}}\right|+\frac{\left|\sigma_{n}-\sigma_{n-1}\right|}{\beta_{n} \theta_{n}}\right\}=0 .
\end{aligned}
$$

Thus, by applying Lemma 2.3 to (3.7), we conclude that

$$
\lim _{n \rightarrow \infty} \frac{\left\|x_{n+1}-x_{n}\right\|}{\theta_{n}}=0
$$

which implies that

$$
\lim _{n \rightarrow \infty}\left\|x_{n+1}-x_{n}\right\|=0
$$

STEP 2. $\lim _{n \rightarrow \infty}\left\|x_{n}-z_{n}\right\|=0$.

Indeed, let $p \in \operatorname{Fix}(T) \cap \operatorname{Fix}(\Gamma) \cap \Xi$. Then we have

$$
\begin{aligned}
\left\|\tilde{y}_{n}-p\right\|= & \left\|P_{C}\left(I-\lambda \nabla f_{\alpha_{n}}\right) y_{n}-P_{C}(I-\lambda \nabla f) p\right\| \\
\leq & \left\|P_{C}\left(I-\lambda \nabla f_{\alpha_{n}}\right) y_{n}-P_{C}\left(I-\lambda \nabla f_{\alpha_{n}}\right) p\right\| \\
& \quad\left\|P_{C}\left(I-\lambda \nabla f_{\alpha_{n}}\right) p-P_{C}(I-\lambda \nabla f) p\right\| \\
\leq & \left\|y_{n}-p\right\|+\left\|P_{C}\left(I-\lambda \nabla f_{\alpha_{n}}\right) p-P_{C}(I-\lambda \nabla f) p\right\| \\
\leq & \left\|y_{n}-p\right\|+\lambda \alpha_{n}\|p\| .
\end{aligned}
$$


Similarly, we get

$$
\left\|\tilde{z}_{n}-p\right\| \leq\left\|z_{n}-p\right\|+\lambda \alpha_{n}\|p\|
$$

By Lemma 2.5 and (3.9), we have

$$
\begin{aligned}
\| z_{n} & -p \|^{2} \\
& =\left\|\beta_{n}\left(Q y_{n}-p\right)+\left(1-\beta_{n}\right)\left(T \tilde{y}_{n}-p\right)\right\|^{2} \\
& \leq \beta_{n}\left\|Q y_{n}-p\right\|^{2}+\left(1-\beta_{n}\right)\left\|\tilde{y}_{n}-p\right\|^{2} \\
& \leq \beta_{n}\left\|Q y_{n}-p\right\|^{2}+\left\|\tilde{y}_{n}-p\right\|^{2} \\
& \leq \beta_{n}\left\|Q y_{n}-p\right\|^{2}+\left(\left\|y_{n}-p\right\|+\lambda \alpha_{n}\|p\|\right)^{2} \\
& =\beta_{n}\left\|Q y_{n}-p\right\|^{2}+\left\|y_{n}-p\right\|^{2}+\lambda \alpha_{n}\|p\|\left(2\left\|y_{n}-p\right\|+\lambda \alpha_{n}\|p\|\right) \\
& \leq \beta_{n}\left\|Q y_{n}-p\right\|^{2}+\theta_{n}\left\|S x_{n}-p\right\|^{2}+\left(1-\theta_{n}\right)\left\|x_{n}-p\right\|^{2}+\lambda \alpha_{n}\|p\|\left(2\left\|y_{n}-p\right\|+\lambda \alpha_{n}\|p\|\right) \\
& \leq \beta_{n}\left\|Q y_{n}-p\right\|^{2}+\theta_{n}\left\|S x_{n}-p\right\|^{2}+\left\|x_{n}-p\right\|^{2}+\lambda \alpha_{n}\|p\|\left(2\left\|y_{n}-p\right\|+\lambda \alpha_{n}\|p\|\right) .
\end{aligned}
$$

Since $\left(\gamma_{n}+\delta_{n}\right) \zeta \leq \gamma_{n}$ for all $n \geq 0$, utilizing Lemma 2.4, we obtain

$$
\begin{aligned}
& \left\|x_{n+1}-p\right\|^{2} \\
& =\left\|\sigma_{n}\left(z_{n}-p\right)+\gamma_{n}\left(\tilde{z}_{n}-p\right)+\delta_{n}\left(\Gamma \tilde{z}_{n}-p\right)\right\|^{2} \\
& =\left\|\sigma_{n}\left(z_{n}-p\right)+\left(\gamma_{n}+\delta_{n}\right) \frac{1}{\gamma_{n}+\delta_{n}}\left[\gamma_{n}\left(\tilde{z}_{n}-p\right)+\delta_{n}\left(\Gamma \tilde{z}_{n}-p\right)\right]\right\|^{2} \\
& =\sigma_{n}\left\|z_{n}-p\right\|^{2}+\left(\gamma_{n}+\delta_{n}\right)\left\|\frac{1}{\gamma_{n}+\delta_{n}}\left[\gamma_{n}\left(\tilde{z}_{n}-p\right)+\delta_{n}\left(\Gamma \tilde{z}_{n}-p\right)\right]\right\|^{2} \\
& -\sigma_{n}\left(\gamma_{n}+\delta_{n}\right)\left\|\left(z_{n}-p\right)-\frac{1}{\gamma_{n}+\delta_{n}}\left[\gamma_{n}\left(\tilde{z}_{n}-p\right)+\delta_{n}\left(\Gamma \tilde{z}_{n}-p\right)\right]\right\|^{2} \\
& =\sigma_{n}\left\|z_{n}-p\right\|^{2}+\left(\gamma_{n}+\delta_{n}\right)\left\|\frac{1}{\gamma_{n}+\delta_{n}}\left[\gamma_{n}\left(\tilde{z}_{n}-p\right)+\delta_{n}\left(\Gamma \tilde{z}_{n}-p\right)\right]\right\|^{2} \\
& -\sigma_{n}\left(\gamma_{n}+\delta_{n}\right)\left\|\frac{1}{\gamma_{n}+\delta_{n}}\left[\gamma_{n}\left(\tilde{z}_{n}-z_{n}\right)+\delta_{n}\left(\Gamma \tilde{z}_{n}-z_{n}\right)\right]\right\|^{2} \\
& =\sigma_{n}\left\|z_{n}-p\right\|^{2}+\left(\gamma_{n}+\delta_{n}\right)\left\|\frac{1}{\gamma_{n}+\delta_{n}}\left[\gamma_{n}\left(\tilde{z}_{n}-p\right)+\delta_{n}\left(\Gamma \tilde{z}_{n}-p\right)\right]\right\|^{2} \\
& -\frac{\sigma_{n}}{\gamma_{n}+\delta_{n}}\left\|x_{n+1}-z_{n}\right\|^{2} \\
& \leq \sigma_{n}\left\|z_{n}-p\right\|^{2}+\left(\gamma_{n}+\delta_{n}\right)\left\|\tilde{z}_{n}-p\right\|^{2}-\frac{\sigma_{n}}{\gamma_{n}+\delta_{n}}\left\|x_{n+1}-z_{n}\right\|^{2} \\
& =\sigma_{n}\left\|z_{n}-p\right\|^{2}+\left(1-\sigma_{n}\right)\left\|\tilde{z}_{n}-p\right\|^{2}-\frac{\sigma_{n}}{1-\sigma_{n}}\left\|x_{n+1}-z_{n}\right\|^{2} \\
& \leq \sigma_{n}\left\|z_{n}-p\right\|^{2}+\left(1-\sigma_{n}\right)\left[\left\|z_{n}-p\right\|^{2}+\lambda \alpha_{n}\|p\|\left(2\left\|z_{n}-p\right\|+\lambda \alpha_{n}\|p\|\right)\right] \\
& -\frac{\sigma_{n}}{1-\sigma_{n}}\left\|x_{n+1}-z_{n}\right\|^{2} \\
& \leq\left\|z_{n}-p\right\|^{2}+\lambda \alpha_{n}\|p\|\left(2\left\|z_{n}-p\right\|+\lambda \alpha_{n}\|p\|\right)-\frac{\sigma_{n}}{1-\sigma_{n}}\left\|x_{n+1}-z_{n}\right\|^{2}
\end{aligned}
$$




$$
\begin{aligned}
\leq & \beta_{n}\left\|Q y_{n}-p\right\|^{2}+\theta_{n}\left\|S x_{n}-p\right\|^{2}+\left\|x_{n}-p\right\|^{2}+\lambda \alpha_{n}\|p\|\left(2\left\|y_{n}-p\right\|+\lambda \alpha_{n}\|p\|\right) \\
& +\lambda \alpha_{n}\|p\|\left(2\left\|z_{n}-p\right\|+\lambda \alpha_{n}\|p\|\right)-\frac{\sigma_{n}}{1-\sigma_{n}}\left\|x_{n+1}-z_{n}\right\|^{2} \\
= & \left\|x_{n}-p\right\|^{2}+\beta_{n}\left\|Q y_{n}-p\right\|^{2}+\theta_{n}\left\|S x_{n}-p\right\|^{2} \\
& +2 \lambda \alpha_{n}\|p\|\left(\left\|y_{n}-p\right\|+\left\|z_{n}-p\right\|+\lambda \alpha_{n}\|p\|\right) \\
& -\frac{\sigma_{n}}{1-\sigma_{n}}\left\|x_{n+1}-z_{n}\right\|^{2} .
\end{aligned}
$$

Since $0<\liminf _{n \rightarrow \infty} \sigma_{n} \leq \limsup _{n \rightarrow \infty} \sigma_{n}<1$, we may assume that $\left\{\sigma_{n}\right\} \subset[c, d]$ for some $c, d \in(0,1)$. Therefore, we deduce

$$
\begin{aligned}
\frac{c}{1-c} & \left\|x_{n+1}-z_{n}\right\|^{2} \\
\leq & \frac{\sigma_{n}}{1-\sigma_{n}}\left\|x_{n+1}-z_{n}\right\|^{2} \\
\leq & \left\|x_{n}-p\right\|^{2}-\left\|x_{n+1}-p\right\|^{2}+\beta_{n}\left\|Q y_{n}-p\right\|^{2}+\theta_{n}\left\|S x_{n}-p\right\|^{2} \\
& +2 \lambda \alpha_{n}\|p\|\left(\left\|y_{n}-p\right\|+\left\|z_{n}-p\right\|+\lambda \alpha_{n}\|p\|\right) \\
\leq & \left(\left\|x_{n}-p\right\|+\left\|x_{n+1}-p\right\|\right)\left\|x_{n}-x_{n+1}\right\|+\beta_{n}\left\|Q y_{n}-p\right\|^{2}+\theta_{n}\left\|S x_{n}-p\right\|^{2} \\
& +2 \lambda \alpha_{n}\|p\|\left(\left\|y_{n}-p\right\|+\left\|z_{n}-p\right\|+\lambda \alpha_{n}\|p\|\right) .
\end{aligned}
$$

Since $\alpha_{n} \rightarrow 0, \beta_{n} \rightarrow 0, \theta_{n} \rightarrow 0$ and $\left\|x_{n}-x_{n+1}\right\| \rightarrow 0$ as $n \rightarrow \infty$, we conclude from the boundedness of $\left\{x_{n}\right\},\left\{y_{n}\right\}$ and $\left\{z_{n}\right\}$ that $\left\|x_{n+1}-z_{n}\right\| \rightarrow 0$ as $n \rightarrow \infty$. This together with $\left\|x_{n}-x_{n+1}\right\| \rightarrow 0$ implies that

$$
\lim _{n \rightarrow \infty}\left\|x_{n}-z_{n}\right\|=0
$$

STEP 3. $\lim _{n \rightarrow \infty}\left\|y_{n}-\tilde{y}_{n}\right\|=0$ and $\lim _{n \rightarrow \infty}\left\|z_{n}-\tilde{z}_{n}\right\|=0$.

Let $p \in \operatorname{Fix}(T) \cap \operatorname{Fix}(\Gamma) \cap \Xi$. Then, by Lemmas 2.2 and 2.5, we have

$$
\begin{aligned}
\| z_{n}- & p \|^{2} \\
= & \left\|\beta_{n}\left(Q y_{n}-p\right)+\left(1-\beta_{n}\right)\left(T \tilde{y}_{n}-p\right)\right\|^{2} \\
\leq & \beta_{n}\left\|Q y_{n}-p\right\|^{2}+\left(1-\beta_{n}\right)\left\|\tilde{y}_{n}-p\right\|^{2} \\
= & \beta_{n}\left\|Q y_{n}-p\right\|^{2}+\left(1-\beta_{n}\right)\left\|P_{C}\left(I-\lambda \nabla f_{\alpha_{n}}\right) y_{n}-P_{C}(I-\lambda \nabla f) p\right\|^{2} \\
\leq & \beta_{n}\left\|Q y_{n}-p\right\|^{2}+\left(1-\beta_{n}\right)\left\|(I-\lambda \nabla f) y_{n}-(I-\lambda \nabla f) p-\lambda \alpha_{n} y_{n}\right\|^{2} \\
\leq & \beta_{n}\left\|Q y_{n}-p\right\|^{2}+\left(1-\beta_{n}\right)\left[\left\|(I-\lambda \nabla f) y_{n}-(I-\lambda \nabla f) p\right\|^{2}\right. \\
& \left.-2 \lambda \alpha_{n}\left\langle y_{n},\left(I-\lambda \nabla f_{\alpha_{n}}\right) y_{n}-(I-\lambda \nabla f) p\right\rangle\right] \\
\leq & \beta_{n}\left\|Q y_{n}-p\right\|^{2}+\left(1-\beta_{n}\right)\left[\left\|y_{n}-p\right\|^{2}+\lambda\left(\lambda-\frac{2}{L}\right)\left\|\nabla f\left(y_{n}\right)-\nabla f(p)\right\|^{2}\right. \\
& \left.+2 \lambda \alpha_{n}\left\|y_{n}\right\|\left\|\left(I-\lambda \nabla f_{\alpha_{n}}\right) y_{n}-(I-\lambda \nabla f) p\right\|\right] \\
\leq & \beta_{n}\left\|Q y_{n}-p\right\|^{2}+\left(1-\beta_{n}\right)\left[\theta_{n}\left\|S x_{n}-p\right\|^{2}+\left(1-\theta_{n}\right)\left\|x_{n}-p\right\|^{2}\right.
\end{aligned}
$$




$$
\begin{aligned}
& \left.+\lambda\left(\lambda-\frac{2}{L}\right)\left\|\nabla f\left(y_{n}\right)-\nabla f(p)\right\|^{2}+2 \lambda \alpha_{n}\left\|y_{n}\right\|\left\|\left(I-\lambda \nabla f_{\alpha_{n}}\right) y_{n}-(I-\lambda \nabla f) p\right\|\right] \\
\leq & \beta_{n}\left\|Q y_{n}-p\right\|^{2}+\theta_{n}\left\|S x_{n}-p\right\|^{2}+\left\|x_{n}-p\right\|^{2}+\left(1-\beta_{n}\right) \lambda\left(\lambda-\frac{2}{L}\right)\left\|\nabla f\left(y_{n}\right)-\nabla f(p)\right\|^{2} \\
& +2 \lambda \alpha_{n}\left\|y_{n}\right\|\left\|\left(I-\lambda \nabla f_{\alpha_{n}}\right) y_{n}-(I-\lambda \nabla f) p\right\| .
\end{aligned}
$$

Therefore, we obtain

$$
\begin{aligned}
(1- & \left.\beta_{n}\right) \lambda\left(\frac{2}{L}-\lambda\right)\left\|\nabla f\left(y_{n}\right)-\nabla f(p)\right\|^{2} \\
\leq & \beta_{n}\left\|Q y_{n}-p\right\|^{2}+\theta_{n}\left\|S x_{n}-p\right\|^{2}+\left\|x_{n}-p\right\|^{2}-\left\|z_{n}-p\right\|^{2} \\
& +2 \lambda \alpha_{n}\left\|y_{n}\right\|\left\|\left(I-\lambda \nabla f_{\alpha_{n}}\right) y_{n}-(I-\lambda \nabla f) p\right\| \\
\leq & \beta_{n}\left\|Q y_{n}-p\right\|^{2}+\theta_{n}\left\|S x_{n}-p\right\|^{2}+\left(\left\|x_{n}-p\right\|+\left\|z_{n}-p\right\|\right)\left(\left\|x_{n}-p\right\|-\left\|z_{n}-p\right\|\right) \\
& +2 \lambda \alpha_{n}\left\|y_{n}\right\|\left\|\left(I-\lambda \nabla f_{\alpha_{n}}\right) y_{n}-(I-\lambda \nabla f) p\right\| \\
\leq & \beta_{n}\left\|Q y_{n}-p\right\|^{2}+\theta_{n}\left\|S x_{n}-p\right\|^{2}+\left(\left\|x_{n}-p\right\|+\left\|z_{n}-p\right\|\right)\left\|x_{n}-z_{n}\right\| \\
& +2 \lambda \alpha_{n}\left\|y_{n}\right\|\left\|\left(I-\lambda \nabla f_{\alpha_{n}}\right) y_{n}-(I-\lambda \nabla f) p\right\| .
\end{aligned}
$$

Since $\alpha_{n} \rightarrow 0, \beta_{n} \rightarrow 0, \theta_{n} \rightarrow 0,\left\|x_{n}-z_{n}\right\| \rightarrow 0$ and $0<\lambda<\frac{2}{L}$, from the boundedness of $\left\{x_{n}\right\},\left\{y_{n}\right\}$ and $\left\{z_{n}\right\}$, we obtain $\lim _{n \rightarrow \infty}\left\|\nabla f\left(y_{n}\right)-\nabla f(p)\right\|=0$, and hence

$$
\lim _{n \rightarrow \infty}\left\|\nabla f_{\alpha_{n}}\left(y_{n}\right)-\nabla f(p)\right\|=0
$$

Also, since

$$
\left\|y_{n}-z_{n}\right\| \leq\left\|y_{n}-x_{n}\right\|+\left\|x_{n}-z_{n}\right\|=\theta_{n}\left\|S x_{n}-x_{n}\right\|+\left\|x_{n}-z_{n}\right\|,
$$

from $\theta_{n} \rightarrow 0$ and $\left\|x_{n}-z_{n}\right\| \rightarrow 0$, it follows that

$$
\lim _{n \rightarrow \infty}\left\|y_{n}-z_{n}\right\|=0 \quad \text { and } \quad \lim _{n \rightarrow \infty}\left\|\nabla f_{\alpha_{n}}\left(z_{n}\right)-\nabla f(p)\right\|=0
$$

Furthermore, from the firm nonexpansiveness of $P_{C}$, we obtain

$$
\begin{aligned}
\left\|\tilde{y}_{n}-p\right\|^{2}= & \left\|P_{C}\left(I-\lambda \nabla f_{\alpha_{n}}\right) y_{n}-P_{C}(I-\lambda \nabla f) p\right\|^{2} \\
\leq & \left\{\left(I-\lambda \nabla f_{\alpha_{n}}\right) y_{n}-(I-\lambda \nabla f) p, \tilde{y}_{n}-p\right\rangle \\
= & \frac{1}{2}\left\{\left\|\left(I-\lambda \nabla f_{\alpha_{n}}\right) y_{n}-(I-\lambda \nabla f) p\right\|^{2}+\left\|\tilde{y}_{n}-p\right\|^{2}\right. \\
& \left.-\left\|\left(I-\lambda \nabla f_{\alpha_{n}}\right) y_{n}-(I-\lambda \nabla f) p-\left(\tilde{y}_{n}-p\right)\right\|^{2}\right\} \\
\leq & \frac{1}{2}\left\{\left\|y_{n}-p\right\|^{2}+2 \lambda\left\|\nabla f_{\alpha_{n}}\left(y_{n}\right)-\nabla f(p)\right\|\left\|\left(I-\lambda \nabla f_{\alpha_{n}}\right) y_{n}-(I-\lambda \nabla f) p\right\|\right. \\
& +\left\|\tilde{y}_{n}-p\right\|^{2}-\left\|y_{n}-\tilde{y}_{n}\right\|^{2}+2 \lambda\left\langle y_{n}-\tilde{y}_{n}, \nabla f_{\alpha_{n}}\left(y_{n}\right)-\nabla f(p)\right\rangle \\
& \left.-\lambda^{2}\left\|\nabla f_{\alpha_{n}}\left(y_{n}\right)-\nabla f(p)\right\|^{2}\right\},
\end{aligned}
$$


and so,

$$
\begin{aligned}
\| \tilde{y}_{n}- & p \|^{2} \\
\leq & \left\|y_{n}-p\right\|^{2}-\left\|y_{n}-\tilde{y}_{n}\right\|^{2} \\
& +2 \lambda\left\|\nabla f_{\alpha_{n}}\left(y_{n}\right)-\nabla f(p)\right\|\left\|\left(I-\lambda \nabla f_{\alpha_{n}}\right) y_{n}-(I-\lambda \nabla f) p\right\| \\
& +2 \lambda\left\langle y_{n}-\tilde{y}_{n}, \nabla f_{\alpha_{n}}\left(y_{n}\right)-\nabla f(p)\right\rangle-\lambda^{2}\left\|\nabla f_{\alpha_{n}}\left(y_{n}\right)-\nabla f(p)\right\|^{2} .
\end{aligned}
$$

Similarly, we have

$$
\begin{aligned}
& \left\|\tilde{z}_{n}-p\right\|^{2} \\
& \leq\left\|z_{n}-p\right\|^{2}-\left\|z_{n}-\tilde{z}_{n}\right\|^{2}+2 \lambda\left\|\nabla f_{\alpha_{n}}\left(z_{n}\right)-\nabla f(p)\right\|\left\|\left(I-\lambda \nabla f_{\alpha_{n}}\right) z_{n}-(I-\lambda \nabla f) p\right\| \\
& +2 \lambda\left\langle z_{n}-\tilde{z}_{n}, \nabla f_{\alpha_{n}}\left(z_{n}\right)-\nabla f(p)\right\rangle-\lambda^{2}\left\|\nabla f_{\alpha_{n}}\left(z_{n}\right)-\nabla f(p)\right\|^{2} .
\end{aligned}
$$

Thus, we have

$$
\begin{aligned}
\left\|z_{n}-p\right\|^{2} \leq & \beta_{n}\left\|Q y_{n}-p\right\|^{2}+\left(1-\beta_{n}\right)\left\|\tilde{y}_{n}-p\right\|^{2} \\
\leq & \beta_{n}\left\|Q y_{n}-p\right\|^{2}+\left\|\tilde{y}_{n}-p\right\|^{2} \\
\leq & \beta_{n}\left\|Q y_{n}-p\right\|^{2}+\left\|y_{n}-p\right\|^{2}-\left\|y_{n}-\tilde{y}_{n}\right\|^{2} \\
& +2 \lambda\left\|\nabla f_{\alpha_{n}}\left(y_{n}\right)-\nabla f(p)\right\|\left\|\left(I-\lambda \nabla f_{\alpha_{n}}\right) y_{n}-(I-\lambda \nabla f) p\right\| \\
& +2 \lambda\left\langle y_{n}-\tilde{y}_{n}, \nabla f_{\alpha_{n}}\left(y_{n}\right)-\nabla f(p)\right\rangle-\lambda^{2}\left\|\nabla f_{\alpha_{n}}\left(y_{n}\right)-\nabla f(p)\right\|^{2} \\
\leq & \beta_{n}\left\|Q y_{n}-p\right\|^{2}+\left\|y_{n}-p\right\|^{2}-\left\|y_{n}-\tilde{y}_{n}\right\|^{2} \\
& +2 \lambda\left\|\nabla f_{\alpha_{n}}\left(y_{n}\right)-\nabla f(p)\right\|\left\|\left(I-\lambda \nabla f_{\alpha_{n}}\right) y_{n}-(I-\lambda \nabla f) p\right\| \\
& +2 \lambda\left\langle y_{n}-\tilde{y}_{n}, \nabla f_{\alpha_{n}}\left(y_{n}\right)-\nabla f(p)\right\rangle \\
\leq & \beta_{n}\left\|Q y_{n}-p\right\|^{2}+\left\|y_{n}-p\right\|^{2}-\left\|y_{n}-\tilde{y}_{n}\right\|^{2} \\
& +2 \lambda\left\|\nabla f_{\alpha_{n}}\left(y_{n}\right)-\nabla f(p)\right\|\left(\left\|\left(I-\lambda \nabla f_{\alpha_{n}}\right) y_{n}-(I-\lambda \nabla f) p\right\|+\left\|y_{n}-\tilde{y}_{n}\right\|\right),
\end{aligned}
$$

which implies that

$$
\begin{aligned}
\| y_{n}- & \tilde{y}_{n} \|^{2} \\
\leq & \beta_{n}\left\|Q y_{n}-p\right\|^{2}+\left\|y_{n}-p\right\|^{2}-\left\|z_{n}-p\right\|^{2} \\
& +2 \lambda\left\|\nabla f_{\alpha_{n}}\left(y_{n}\right)-\nabla f(p)\right\|\left(\left\|\left(I-\lambda \nabla f_{\alpha_{n}}\right) y_{n}-(I-\lambda \nabla f) p\right\|+\left\|y_{n}-\tilde{y}_{n}\right\|\right) \\
\leq & \beta_{n}\left\|Q y_{n}-p\right\|^{2}+\left(\left\|y_{n}-p\right\|+\left\|z_{n}-p\right\|\right)\left\|y_{n}-z_{n}\right\| \\
& +2 \lambda\left\|\nabla f_{\alpha_{n}}\left(y_{n}\right)-\nabla f(p)\right\|\left(\left\|\left(I-\lambda \nabla f_{\alpha_{n}}\right) y_{n}-(I-\lambda \nabla f) p\right\|+\left\|y_{n}-\tilde{y}_{n}\right\|\right) .
\end{aligned}
$$

Since $\beta_{n} \rightarrow 0,\left\|y_{n}-z_{n}\right\| \rightarrow 0$ and $\left\|\nabla f_{\alpha_{n}}\left(y_{n}\right)-\nabla f(p)\right\| \rightarrow 0$, from the boundedness of $\left\{x_{n}\right\}$, $\left\{y_{n}\right\},\left\{z_{n}\right\}$ and $\left\{\tilde{y}_{n}\right\}$, it follows that

$$
\lim _{n \rightarrow \infty}\left\|y_{n}-\tilde{y}_{n}\right\|=0
$$


In addition, since $\left(\gamma_{n}+\delta_{n}\right) \zeta \leq \gamma_{n}$ for all $n \geq 0$, utilizing Lemma 2.4, we get from (3.12)

$$
\begin{aligned}
\left\|x_{n+1}-p\right\|^{2} \leq & \sigma_{n}\left\|z_{n}-p\right\|^{2}+\left(\gamma_{n}+\delta_{n}\right)\left\|\tilde{z}_{n}-p\right\|^{2} \\
= & \sigma_{n}\left\|z_{n}-p\right\|^{2}+\left(1-\sigma_{n}\right)\left\|\tilde{z}_{n}-p\right\|^{2} \\
\leq & \sigma_{n}\left\|z_{n}-p\right\|^{2}+\left(1-\sigma_{n}\right)\left\{\left\|z_{n}-p\right\|^{2}-\left\|z_{n}-\tilde{z}_{n}\right\|^{2}\right. \\
& +2 \lambda\left\|\nabla f_{\alpha_{n}}\left(z_{n}\right)-\nabla f(p)\right\|\left\|\left(I-\lambda \nabla f_{\alpha_{n}}\right) z_{n}-(I-\lambda \nabla f) p\right\| \\
& \left.+2 \lambda\left\langle z_{n}-\tilde{z}_{n}, \nabla f_{\alpha_{n}}\left(z_{n}\right)-\nabla f(p)\right\rangle-\lambda^{2}\left\|\nabla f_{\alpha_{n}}\left(z_{n}\right)-\nabla f(p)\right\|^{2}\right\} \\
\leq & \sigma_{n}\left\|z_{n}-p\right\|^{2}+\left(1-\sigma_{n}\right)\left\{\left\|z_{n}-p\right\|^{2}-\left\|z_{n}-\tilde{z}_{n}\right\|^{2}\right. \\
& +2 \lambda\left\|\nabla f_{\alpha_{n}}\left(z_{n}\right)-\nabla f(p)\right\|\left\|\left(I-\lambda \nabla f_{\alpha_{n}}\right) z_{n}-(I-\lambda \nabla f) p\right\| \\
& \left.+2 \lambda\left\|z_{n}-\tilde{z}_{n}\right\|\left\|\nabla f_{\alpha_{n}}\left(z_{n}\right)-\nabla f(p)\right\|\right\} \\
\leq & \left\|z_{n}-p\right\|^{2}-\left(1-\sigma_{n}\right)\left\|z_{n}-\tilde{z}_{n}\right\|^{2} \\
& +2 \lambda\left\|\nabla f_{\alpha_{n}}\left(z_{n}\right)-\nabla f(p)\right\|\left(\left\|\left(I-\lambda \nabla f_{\alpha_{n}}\right) z_{n}-(I-\lambda \nabla f) p\right\|+\left\|z_{n}-\tilde{z}_{n}\right\|\right)
\end{aligned}
$$

which implies that

$$
\begin{aligned}
(1- & \left.\sigma_{n}\right)\left\|z_{n}-\tilde{z}_{n}\right\|^{2} \\
\leq & \left\|z_{n}-p\right\|^{2}-\left\|x_{n+1}-p\right\|^{2} \\
& +2 \lambda\left\|\nabla f_{\alpha_{n}}\left(z_{n}\right)-\nabla f(p)\right\|\left(\left\|\left(I-\lambda \nabla f_{\alpha_{n}}\right) z_{n}-(I-\lambda \nabla f) p\right\|+\left\|z_{n}-\tilde{z}_{n}\right\|\right) \\
& \leq\left(\left\|z_{n}-p\right\|+\left\|x_{n+1}-p\right\|\right)\left\|z_{n}-x_{n+1}\right\| \\
& +2 \lambda\left\|\nabla f_{\alpha_{n}}\left(z_{n}\right)-\nabla f(p)\right\|\left(\left\|\left(I-\lambda \nabla f_{\alpha_{n}}\right) z_{n}-(I-\lambda \nabla f) p\right\|+\left\|z_{n}-\tilde{z}_{n}\right\|\right) .
\end{aligned}
$$

Since $\left\{\sigma_{n}\right\} \subset[c, d],\left\|z_{n}-x_{n+1}\right\| \rightarrow 0$ and $\left\|\nabla f_{\alpha_{n}}\left(z_{n}\right)-\nabla f(p)\right\| \rightarrow 0$, from the boundedness of $\left\{x_{n}\right\},\left\{z_{n}\right\}$ and $\left\{\tilde{z}_{n}\right\}$, it follows that

$$
\lim _{n \rightarrow \infty}\left\|z_{n}-\tilde{z}_{n}\right\|=0
$$

STEP 4. $\omega_{w}\left(x_{n}\right) \subset \Omega$.

Let $p^{*} \in \omega_{w}\left(x_{n}\right)$. Then there exists a subsequence $\left\{x_{n_{i}}\right\}$ of $\left\{x_{n}\right\}$ such that $x_{n_{i}} \rightarrow p^{*}$. Since

$$
\begin{aligned}
z_{n}-y_{n} & =\beta_{n}\left(Q y_{n}-y_{n}\right)+\left(1-\beta_{n}\right)\left(T \tilde{y}_{n}-y_{n}\right) \\
& =\beta_{n}\left(Q y_{n}-y_{n}\right)+\left(1-\beta_{n}\right)\left(T \tilde{y}_{n}-\tilde{y}_{n}\right)+\left(1-\beta_{n}\right)\left(\tilde{y}_{n}-y_{n}\right),
\end{aligned}
$$

we have

$$
\begin{aligned}
\left(1-\beta_{n}\right)\left\|T \tilde{y}_{n}-\tilde{y}_{n}\right\| & =\left\|z_{n}-y_{n}-\beta_{n}\left(Q y_{n}-y_{n}\right)-\left(1-\beta_{n}\right)\left(\tilde{y}_{n}-y_{n}\right)\right\| \\
& \leq\left\|z_{n}-y_{n}\right\|+\beta_{n}\left\|Q y_{n}-y_{n}\right\|+\left(1-\beta_{n}\right)\left\|\tilde{y}_{n}-y_{n}\right\| \\
& \leq\left\|z_{n}-y_{n}\right\|+\beta_{n}\left\|Q y_{n}-y_{n}\right\|+\left\|\tilde{y}_{n}-y_{n}\right\| .
\end{aligned}
$$

Hence from $\left\|z_{n}-y_{n}\right\| \rightarrow 0, \beta_{n} \rightarrow 0$ and $\left\|\tilde{y}_{n}-y_{n}\right\| \rightarrow 0$, we get $\lim _{n \rightarrow \infty}\left\|T \tilde{y}_{n}-\tilde{y}_{n}\right\|=0$. Since $\left\|x_{n}-y_{n}\right\| \rightarrow 0$ and $\left\|y_{n}-\tilde{y}_{n}\right\| \rightarrow 0$, we have $\tilde{y}_{n_{i}} \rightarrow p^{*}$. By Lemma 2.1(b) (demiclosedness principle), we obtain $p^{*} \in \operatorname{Fix}(T)$. 
Meanwhile, observe that

$$
\begin{aligned}
x_{n+1}-z_{n} & =\gamma_{n}\left(\tilde{z}_{n}-z_{n}\right)+\delta_{n}\left(\Gamma \tilde{z}_{n}-\tilde{z}_{n}\right)+\delta_{n}\left(\tilde{z}_{n}-z_{n}\right) \\
& =\left(\gamma_{n}+\delta_{n}\right)\left(\tilde{z}_{n}-z_{n}\right)+\delta_{n}\left(\Gamma \tilde{z}_{n}-\tilde{z}_{n}\right) \\
& =\left(1-\sigma_{n}\right)\left(\tilde{z}_{n}-z_{n}\right)+\delta_{n}\left(\Gamma \tilde{z}_{n}-\tilde{z}_{n}\right) .
\end{aligned}
$$

Thus,

$$
\begin{aligned}
\delta_{n}\left\|\Gamma \tilde{z}_{n}-\tilde{z}_{n}\right\| & =\left\|x_{n+1}-z_{n}-\left(1-\sigma_{n}\right)\left(\tilde{z}_{n}-z_{n}\right)\right\| \\
& \leq\left\|x_{n+1}-z_{n}\right\|+\left(1-\sigma_{n}\right)\left\|\tilde{z}_{n}-z_{n}\right\| \\
& \leq\left\|x_{n+1}-z_{n}\right\|+\left\|\tilde{z}_{n}-z_{n}\right\| \rightarrow 0 \quad \text { as } n \rightarrow \infty .
\end{aligned}
$$

This together with $\liminf _{n \rightarrow \infty} \delta_{n}>0$ yields $\lim _{n \rightarrow \infty}\left\|\Gamma \tilde{z}_{n}-\tilde{z}_{n}\right\|=0$. Since $\left\|x_{n}-z_{n}\right\| \rightarrow 0$ and $\left\|z_{n}-\tilde{z}_{n}\right\| \rightarrow 0$, we have $\tilde{z}_{n_{i}} \rightarrow p^{*}$. By Lemma 2.1(b) (demiclosedness principle), we have $p^{*} \in \operatorname{Fix}(\Gamma)$.

Further, let us show $p^{*} \in \Xi$. Indeed, from $\left\|x_{n}-y_{n}\right\| \rightarrow 0$ and $\left\|\tilde{y}_{n}-y_{n}\right\| \rightarrow 0$, we have $y_{n_{i}} \rightarrow p^{*}$ and $\tilde{y}_{n_{i}} \rightarrow p^{*}$. Define

$$
V v= \begin{cases}\nabla f(v)+N_{C} v & \text { if } v \in C, \\ \emptyset & \text { if } v \notin C,\end{cases}
$$

where $N_{C} v=\{w \in H:\langle v-u, w\rangle \geq 0, \forall u \in C\}$. Then $V$ is maximal monotone and $0 \in V v$ if and only if $v \in \operatorname{VI}(C, \nabla f)$ (see [17]). Let $(v, w) \in \operatorname{graph}(V)$. Then we have

$$
w \in V v=\nabla f(v)+N_{C} v
$$

and hence

$$
w-\nabla f(v) \in N_{C} v
$$

Therefore, we have

$$
\langle v-u, w-\nabla f(v)\rangle \geq 0, \quad \forall u \in C .
$$

On the other hand, from

$$
\tilde{y}_{n}=P_{C}\left(y_{n}-\lambda \nabla f_{\alpha_{n}}\left(y_{n}\right)\right) \text { and } \quad v \in C \text {, }
$$

we have

$$
\left\langle y_{n}-\lambda \nabla f_{\alpha_{n}}\left(y_{n}\right)-\tilde{y}_{n}, \tilde{y}_{n}-v\right\rangle \geq 0,
$$

and hence

$$
\left\langle v-\tilde{y}_{n}, \frac{\tilde{y}_{n}-y_{n}}{\lambda}+\nabla f_{\alpha_{n}}\left(y_{n}\right)\right\rangle \geq 0 .
$$


Therefore, from

$$
w-\nabla f(v) \in N_{C}(v) \text { and } \quad \tilde{y}_{n_{i}} \in C,
$$

we have

$$
\begin{aligned}
\left\langle v-\tilde{y}_{n_{i}}, w\right\rangle \geq & \left\langle v-\tilde{y}_{n_{i}}, \nabla f(v)\right\rangle \\
\geq & \left\langle v-\tilde{y}_{n_{i}}, \nabla f(v)\right\rangle-\left\langle v-\tilde{y}_{n_{i}}, \frac{\tilde{y}_{n_{i}}-y_{n_{i}}}{\lambda}+\nabla f_{\alpha_{n_{i}}}\left(y_{n_{i}}\right)\right\rangle \\
= & \left\langle v-\tilde{y}_{n_{i}}, \nabla f(v)\right\rangle-\left\langle v-\tilde{y}_{n_{i}}, \frac{\tilde{y}_{n_{i}}-y_{n_{i}}}{\lambda}+\nabla f\left(y_{n_{i}}\right)\right\rangle-\alpha_{n_{i}}\left\langle v-\tilde{y}_{n_{i}}, y_{n_{i}}\right\rangle \\
= & \left\langle v-\tilde{y}_{n_{i}}, \nabla f(v)-\nabla f\left(\tilde{y}_{n_{i}}\right)\right\rangle+\left\langle v-\tilde{y}_{n_{i}}, \nabla f\left(\tilde{y}_{n_{i}}\right)-\nabla f\left(y_{n_{i}}\right)\right\rangle \\
& -\left\langle v-\tilde{y}_{n_{i}}, \frac{\tilde{y}_{n_{i}}-y_{n_{i}}}{\lambda}\right\rangle-\alpha_{n_{i}}\left\langle v-\tilde{y}_{n_{i}}, y_{n_{i}}\right\rangle \\
\geq & \left\langle v-\tilde{y}_{n_{i}}, \nabla f\left(\tilde{y}_{n_{i}}\right)-\nabla f\left(y_{n_{i}}\right)\right\rangle-\left\langle v-\tilde{y}_{n_{i}}, \frac{\tilde{y}_{n_{i}}-y_{n_{i}}}{\lambda}\right\rangle-\alpha_{n_{i}}\left\langle v-\tilde{y}_{n_{i}}, y_{n_{i}}\right\rangle .
\end{aligned}
$$

Hence, we obtain

$$
\left\langle v-p^{*}, w\right\rangle \geq 0 \quad \text { as } i \rightarrow \infty
$$

Since $V$ is maximal monotone, we have $p^{*} \in V^{-1} 0$, and hence $p^{*} \in \operatorname{VI}(C, \nabla f)$, which leads to $p \in \Xi$. Consequently, $p^{*} \in \operatorname{Fix}(T) \cap \operatorname{Fix}(\Gamma) \cap \Xi$. This shows that $\omega_{w}\left(x_{n}\right) \subset \operatorname{Fix}(T) \cap$ $\operatorname{Fix}(\Gamma) \cap \Xi$.

Finally, let us show $p^{*} \in \Omega$. Indeed, it follows from (2.2) that for every $p \in \operatorname{Fix}(T) \cap$ $\operatorname{Fix}(\Gamma) \cap \Xi$,

$$
\begin{aligned}
\left\|y_{n}-p\right\|^{2} & =\left\|\left(1-\theta_{n}\right)\left(x_{n}-p\right)+\theta_{n}\left(S x_{n}-S p\right)+\theta_{n}(S p-p)\right\|^{2} \\
& \leq\left\|\left(1-\theta_{n}\right)\left(x_{n}-p\right)+\theta_{n}\left(S x_{n}-S p\right)\right\|^{2}+2 \theta_{n}\left\langle S p-p, y_{n}-p\right\rangle \\
& \leq\left(1-\theta_{n}\right)\left\|x_{n}-p\right\|^{2}+\theta_{n}\left\|S x_{n}-S p\right\|^{2}+2 \theta_{n}\left\langle S p-p, y_{n}-p\right\rangle \\
& \leq\left\|x_{n}-p\right\|^{2}+2 \theta_{n}\left\langle S p-p, y_{n}-p\right\rangle,
\end{aligned}
$$

and hence

$$
\begin{aligned}
&\left\|z_{n}-p\right\|^{2} \\
& \leq \beta_{n}\left\|Q y_{n}-p\right\|^{2}+\left(1-\beta_{n}\right)\left\|\tilde{y}_{n}-p\right\|^{2} \\
& \leq \beta_{n}\left\|Q y_{n}-p\right\|^{2}+\left\|\tilde{y}_{n}-p\right\|^{2} \\
& \leq \beta_{n}\left\|Q y_{n}-p\right\|^{2}+\left(\left\|y_{n}-p\right\|+\lambda \alpha_{n}\|p\|\right)^{2} \\
& \leq \beta_{n}\left\|Q y_{n}-p\right\|^{2}+\left\|y_{n}-p\right\|^{2}+\lambda \alpha_{n}\|p\|\left(2\left\|y_{n}-p\right\|+\lambda \alpha_{n}\|p\|\right) \\
& \leq \beta_{n}\left\|Q y_{n}-p\right\|^{2}+\left\|x_{n}-p\right\|^{2}+2 \theta_{n}\left\langle S p-p, y_{n}-p\right\rangle \\
&+\lambda \alpha_{n}\|p\|\left(2\left\|y_{n}-p\right\|+\lambda \alpha_{n}\|p\|\right) .
\end{aligned}
$$


Since $\left(\gamma_{n}+\delta_{n}\right) \zeta \leq \gamma_{n}$ for all $n \geq 0$, by Lemma 2.4, we have

$$
\begin{aligned}
&\left\|x_{n+1}-p\right\|^{2} \\
& \leq \sigma_{n}\left\|z_{n}-p\right\|^{2}+\left(\gamma_{n}+\delta_{n}\right)\left\|\tilde{z}_{n}-p\right\|^{2} \\
& \leq \sigma_{n}\left\|z_{n}-p\right\|^{2}+\left(1-\sigma_{n}\right)\left(\left\|z_{n}-p\right\|+\lambda \alpha_{n}\|p\|\right)^{2} \\
& \leq\left\|z_{n}-p\right\|^{2}+\lambda \alpha_{n}\|p\|\left(2\left\|z_{n}-p\right\|+\lambda \alpha_{n}\|p\|\right) \\
& \leq \beta_{n}\left\|Q y_{n}-p\right\|^{2}+\left\|x_{n}-p\right\|^{2}+2 \theta_{n}\left\langle S p-p, y_{n}-p\right\rangle \\
&+\lambda \alpha_{n}\|p\|\left(2\left\|y_{n}-p\right\|+\lambda \alpha_{n}\|p\|\right)+\lambda \alpha_{n}\|p\|\left(2\left\|z_{n}-p\right\|+\lambda \alpha_{n}\|p\|\right) \\
&=\left\|x_{n}-p\right\|^{2}+\beta_{n}\left\|Q y_{n}-p\right\|^{2}+2 \theta_{n}\left\langle S p-p, y_{n}-p\right\rangle \\
&+2 \lambda \alpha_{n}\|p\|\left(\left\|y_{n}-p\right\|+\left\|z_{n}-p\right\|+\lambda \alpha_{n}\|p\|\right),
\end{aligned}
$$

which implies that

$$
\begin{aligned}
2\left\langle p-S p, y_{n}-p\right\rangle \leq & \frac{1}{\theta_{n}}\left(\left\|x_{n}-p\right\|^{2}-\left\|x_{n+1}-p\right\|^{2}\right)+\frac{\beta_{n}}{\theta_{n}}\left\|Q y_{n}-p\right\|^{2} \\
& +\frac{\alpha_{n}}{\theta_{n}} 2 \lambda\|p\|\left(\left\|y_{n}-p\right\|+\left\|z_{n}-p\right\|+\lambda \alpha_{n}\|p\|\right) \\
\leq & \frac{\left\|x_{n}-x_{n+1}\right\|}{\theta_{n}}\left(\left\|x_{n}-p\right\|+\left\|x_{n+1}-p\right\|\right)+\frac{\beta_{n}}{\theta_{n}}\left\|Q y_{n}-p\right\|^{2} \\
& +\frac{\alpha_{n}}{\theta_{n}} 2 \lambda\|p\|\left(\left\|y_{n}-p\right\|+\left\|z_{n}-p\right\|+\lambda \alpha_{n}\|p\|\right) .
\end{aligned}
$$

Since $\frac{\alpha_{n}+\beta_{n}}{\theta_{n}} \rightarrow 0$ and $\frac{\left\|x_{n}-x_{n+1}\right\|}{\theta_{n}} \rightarrow 0$ as $n \rightarrow \infty$, from the boundedness of $\left\{x_{n}\right\},\left\{y_{n}\right\}$ and $\left\{z_{n}\right\}$, we deduce that

$$
\limsup _{n \rightarrow \infty}\left\langle p-S p, y_{n}-p\right\rangle \leq 0, \quad \forall p \in \operatorname{Fix}(T) \cap \operatorname{Fix}(\Gamma) \cap \Xi
$$

So, from $y_{n_{i}} \rightarrow p^{*}$, we get

$$
\left\langle p-S p, p^{*}-p\right\rangle \leq 0, \quad \forall p \in \operatorname{Fix}(T) \cap \operatorname{Fix}(\Gamma) \cap \Xi
$$

Taking into consideration that $I-S$ is monotone and continuous, utilizing Minty's lemma [7], we have

$$
\left\langle p^{*}-S p^{*}, p^{*}-p\right\rangle \leq 0, \quad \forall p \in \operatorname{Fix}(T) \cap \operatorname{Fix}(\Gamma) \cap \Xi
$$

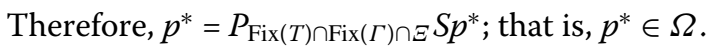

Remark 3.1 Iterative algorithm (2.2) is different from the algorithms in $[1,11]$. The twostep iterative scheme in [11] for two nonexpansive mappings and the gradient-projection iterative schemes in [1] for MP (1.1) are extended to develop three-step iterative scheme (2.2) with regularization for MP (1.1), two nonexpansive mappings and a strictly pseudocontractive mapping. 
Remark 3.2 The following sequences satisfy the hypotheses on the parameter in Theorem 3.1.

(a) $\alpha_{n}=\frac{1}{n^{1+s+t}}, \beta_{n}=\frac{1}{n^{s}}$ and $\theta_{n}=\frac{1}{n^{t}}$, where $t \in\left(0, \frac{1}{2}\right)$ and $s \in(t, 1-t)$;

(b) $\sigma_{n}=\frac{1}{2}+\frac{2}{n}$ and $\gamma_{n}=\delta_{n}=\frac{1}{4}-\frac{1}{n}$ for all $n>4$.

Theorem 3.2 Let $\left\{x_{n}\right\}$ be the bounded sequence generated from any given $x_{0} \in C$ by (2.2). Assume that hypotheses (H1)-(H5) of Theorem 3.1 hold and

(H6) $\lim _{n \rightarrow \infty} \frac{\theta_{n}^{2}}{\beta_{n}}=0$;

(H7) There is a constant $k>0$ such that

$\left\|x-T P_{C}(I-\lambda \nabla f) x\right\| \geq k \operatorname{dist}(x, \operatorname{Fix}(T) \cap \operatorname{Fix}(\Gamma) \cap \Xi)$ for each $x \in C$, where $\operatorname{dist}(x, \operatorname{Fix}(T) \cap \operatorname{Fix}(\Gamma) \cap \Xi)=\inf _{y \in \operatorname{Fix}(T) \cap \operatorname{Fix}(\Gamma) \cap \Xi}\|x-y\|$.

Then the sequences $\left\{x_{n}\right\},\left\{y_{n}\right\}$ and $\left\{z_{n}\right\}$ converge strongly to $x^{*}=P_{\Omega} Q x^{*}$ provided $\left\|x_{n}-z_{n}\right\|=$ $o\left(\theta_{n}\right)$, where $x^{*}$ solves the following variational inequality:

$$
\left\langle x^{*}-S x^{*}, x^{*}-x\right\rangle \leq 0, \quad \forall x \in \operatorname{Fix}(T) \cap \operatorname{Fix}(\Gamma) \cap \Xi .
$$

Proof Let $p \in \operatorname{Fix}(T) \cap \operatorname{Fix}(\Gamma) \cap \Xi$. From (2.2), we have

$$
z_{n}-p=\beta_{n}\left(Q y_{n}-Q p\right)+\beta_{n}(Q p-p)+\left(1-\beta_{n}\right)\left(T \tilde{y}_{n}-p\right),
$$

and therefore,

$$
\begin{aligned}
\| z_{n}- & p \|^{2} \\
\leq & \left\|\beta_{n}\left(Q y_{n}-Q p\right)+\left(1-\beta_{n}\right)\left(T \tilde{y}_{n}-p\right)\right\|^{2}+2 \beta_{n}\left\langle Q p-p, z_{n}-p\right\rangle \\
\leq & \left(1-\beta_{n}\right)\left\|T \tilde{y}_{n}-p\right\|^{2}+\beta_{n}\left\|Q y_{n}-Q p\right\|^{2}+2 \beta_{n}\left\langle Q p-p, z_{n}-p\right\rangle \\
\leq & \left(1-\beta_{n}\right)\left\|\tilde{y}_{n}-p\right\|^{2}+\beta_{n} \rho^{2}\left\|y_{n}-p\right\|^{2}+2 \beta_{n}\left\langle Q p-p, z_{n}-p\right\rangle \\
\leq & \left(1-\beta_{n}\right)\left(\left\|y_{n}-p\right\|+\lambda \alpha_{n}\|p\|\right)^{2}+\beta_{n} \rho\left\|y_{n}-p\right\|^{2}+2 \beta_{n}\left\langle Q p-p, z_{n}-p\right\rangle \\
\leq & \left(1-(1-\rho) \beta_{n}\right)\left\|y_{n}-p\right\|^{2}+\lambda \alpha_{n}\|p\|\left(2\left\|y_{n}-p\right\|+\lambda \alpha_{n}\|p\|\right) \\
& +2 \beta_{n}\left\langle Q p-p, z_{n}-p\right\rangle .
\end{aligned}
$$

Again from (2.2), we obtain

$$
\begin{aligned}
\left\|y_{n}-p\right\|^{2} & =\left\|\left(1-\theta_{n}\right)\left(x_{n}-p\right)+\theta_{n}\left(S x_{n}-S p\right)+\theta_{n}(S p-p)\right\|^{2} \\
& \leq\left\|\left(1-\theta_{n}\right)\left(x_{n}-p\right)+\theta_{n}\left(S x_{n}-S p\right)\right\|^{2}+2 \theta_{n}\left\langle S p-p, y_{n}-p\right\rangle \\
& \leq\left(1-\theta_{n}\right)\left\|x_{n}-p\right\|^{2}+\theta_{n}\left\|S x_{n}-S p\right\|^{2}+2 \theta_{n}\left\langle S p-p, y_{n}-p\right\rangle \\
& \leq\left\|x_{n}-p\right\|^{2}+2 \theta_{n}\left\langle S p-p, y_{n}-p\right\rangle .
\end{aligned}
$$

Substituting (3.14) into (3.13), we get

$$
\begin{aligned}
\left\|z_{n}-p\right\|^{2} \leq & \left(1-(1-\rho) \beta_{n}\right)\left(\left\|x_{n}-p\right\|^{2}+2 \theta_{n}\left\langle S p-p, y_{n}-p\right\rangle\right) \\
& +\lambda \alpha_{n}\|p\|\left(2\left\|y_{n}-p\right\|+\lambda \alpha_{n}\|p\|\right)+2 \beta_{n}\left\langle Q p-p, z_{n}-p\right\rangle
\end{aligned}
$$




$$
\begin{aligned}
= & \left(1-(1-\rho) \beta_{n}\right)\left\|x_{n}-p\right\|^{2}+2\left(1-(1-\rho) \beta_{n}\right) \theta_{n}\left\langle S p-p, y_{n}-p\right\rangle \\
& +2 \beta_{n}\left\langle Q p-p, z_{n}-p\right\rangle+\lambda \alpha_{n}\|p\|\left(2\left\|y_{n}-p\right\|+\lambda \alpha_{n}\|p\|\right) .
\end{aligned}
$$

Since $\left(\gamma_{n}+\delta_{n}\right) \zeta \leq \gamma_{n}$ for all $n \geq 0$, utilizing Lemma 2.4, we get from (2.2) and (3.15)

$$
\begin{aligned}
\left\|x_{n+1}-p\right\|^{2} \leq & \sigma_{n}\left\|z_{n}-p\right\|^{2}+\left(\gamma_{n}+\delta_{n}\right)\left\|\tilde{z}_{n}-p\right\|^{2} \\
\leq & \sigma_{n}\left\|z_{n}-p\right\|^{2}+\left(1-\sigma_{n}\right)\left(\left\|z_{n}-p\right\|+\lambda \alpha_{n}\|p\|\right)^{2} \\
\leq & \left\|z_{n}-p\right\|^{2}+\lambda \alpha_{n}\|p\|\left(2\left\|z_{n}-p\right\|+\lambda \alpha_{n}\|p\|\right) \\
\leq & \left(1-(1-\rho) \beta_{n}\right)\left\|x_{n}-p\right\|^{2}+2\left(1-(1-\rho) \beta_{n}\right) \theta_{n}\left\langle S p-p, y_{n}-p\right\rangle \\
& +2 \beta_{n}\left\langle Q p-p, z_{n}-p\right\rangle+\lambda \alpha_{n}\|p\|\left(2\left\|y_{n}-p\right\|+\lambda \alpha_{n}\|p\|\right) \\
& +\lambda \alpha_{n}\|p\|\left(2\left\|z_{n}-p\right\|+\lambda \alpha_{n}\|p\|\right) \\
= & \left(1-(1-\rho) \beta_{n}\right)\left\|x_{n}-p\right\|^{2}+2\left(1-(1-\rho) \beta_{n}\right) \theta_{n}\left\langle S p-p, y_{n}-p\right\rangle \\
& +2 \beta_{n}\left\langle Q p-p, z_{n}-p\right\rangle+2 \lambda \alpha_{n}\|p\|\left(\left\|y_{n}-p\right\|+\left\|z_{n}-p\right\|+\lambda \alpha_{n}\|p\|\right) \\
\leq & \left(1-(1-\rho) \beta_{n}\right)\left\|x_{n}-p\right\|^{2}+2\left(1-(1-\rho) \beta_{n}\right) \theta_{n}\left\langle S p-p, y_{n}-p\right\rangle \\
& +2 \beta_{n}\left\langle Q p-p, z_{n}-p\right\rangle+\widetilde{M} \alpha_{n},
\end{aligned}
$$

where $\widetilde{M}=\sup _{n \geq 0}\left\{2 \lambda\|p\|\left(\left\|y_{n}-p\right\|+\left\|z_{n}-p\right\|+\lambda \alpha_{n}\|p\|\right)\right\}<\infty$.

Taking into consideration that $P_{\Omega} \circ Q$ is a contractive mapping, we know that $P_{\Omega} \circ Q$ has a unique fixed point $x^{*} \in \Omega$. That is, there is a unique solution $x^{*} \in \Omega$ of the following variational inequality problem (VIP):

$$
\left\langle Q x^{*}-x^{*}, q-x^{*}\right\rangle \leq 0, \quad \forall q \in \Omega .
$$

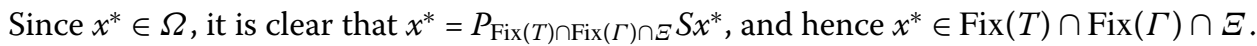
Thus, from (3.16), we conclude that

$$
\begin{aligned}
&\left\|x_{n+1}-x^{*}\right\|^{2} \\
& \leq\left(1-(1-\rho) \beta_{n}\right)\left\|x_{n}-x^{*}\right\|^{2}+2\left(1-(1-\rho) \beta_{n}\right) \theta_{n}\left\langle S x^{*}-x^{*}, y_{n}-x^{*}\right\rangle \\
&+2 \beta_{n}\left\langle Q x^{*}-x^{*}, z_{n}-x^{*}\right\rangle+\widetilde{M} \alpha_{n} \\
&=\left(1-(1-\rho) \beta_{n}\right)\left\|x_{n}-x^{*}\right\|^{2}+(1-\rho) \beta_{n}\left\{\frac{2\left(1-(1-\rho) \beta_{n}\right)}{1-\rho} \frac{\theta_{n}}{\beta_{n}}\left\langle S x^{*}-x^{*}, y_{n}-x^{*}\right\rangle\right. \\
&\left.+\frac{2}{1-\rho}\left\langle Q x^{*}-x^{*}, z_{n}-x^{*}\right\rangle\right\}+\tilde{M} \alpha_{n} .
\end{aligned}
$$

Consider a subsequence $\left\{x_{n_{i}}\right\}$ of $\left\{x_{n}\right\}$ such that

$$
\limsup _{n \rightarrow \infty}\left\langle Q x^{*}-x^{*}, x_{n}-x^{*}\right\rangle=\lim _{i \rightarrow \infty}\left\langle Q x^{*}-x^{*}, x_{n_{i}}-x^{*}\right\rangle
$$

Without loss of generality, we may further assume that $x_{n_{i}}-\tilde{x}$. Then, in view of Theorem 3.1, $\tilde{x} \in \Omega$. Since $x^{*}$ is a unique solution of VIP (3.17) and $\left\|x_{n}-z_{n}\right\| \rightarrow 0$, we 
have

$$
\begin{aligned}
\limsup _{n \rightarrow \infty}\left\langle Q x^{*}-x^{*}, z_{n}-x^{*}\right\rangle & =\limsup _{n \rightarrow \infty}\left(\left\langle Q x^{*}-x^{*}, z_{n}-x_{n}\right\rangle+\left\langle Q x^{*}-x^{*}, x_{n}-x^{*}\right\rangle\right) \\
& =\limsup _{n \rightarrow \infty}\left\langle Q x^{*}-x^{*}, x_{n}-x^{*}\right\rangle=\lim _{i \rightarrow \infty}\left\langle Q x^{*}-x^{*}, x_{n_{i}}-x^{*}\right\rangle \\
& =\left\langle Q x^{*}-x^{*}, \tilde{x}-x^{*}\right\rangle \leq 0,
\end{aligned}
$$

which implies that

$$
\limsup _{n \rightarrow \infty} \frac{2}{1-\rho}\left\langle Q x^{*}-x^{*}, z_{n}-x^{*}\right\rangle \leq 0 .
$$

Meanwhile, from $x^{*} \in \Omega$ and (H7), we infer that

$$
\begin{aligned}
& \left\langle S x^{*}-x^{*}, y_{n}-x^{*}\right\rangle
\end{aligned}
$$

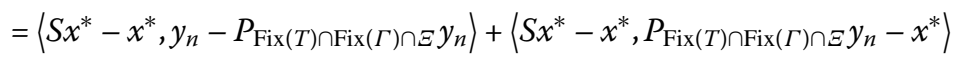

$$
\begin{aligned}
& \leq\left\langle S x^{*}-x^{*}, y_{n}-P_{\left.\operatorname{Fix}(T) \cap \operatorname{Fix}(\Gamma) \cap \Xi y_{n}\right\rangle}\right. \\
& \leq\left\|S x^{*}-x^{*}\right\| \| y_{n}-P_{\mathrm{Fix}(T) \cap \mathrm{Fix}(\Gamma) \cap \Xi y_{n} \|} \\
& =\operatorname{dist}\left(y_{n}, \operatorname{Fix}(T) \cap \operatorname{Fix}(\Gamma) \cap \Xi\right)\left\|S x^{*}-x^{*}\right\| \\
& \leq \frac{1}{k}\left\|S x^{*}-x^{*}\right\|\left\|y_{n}-T P_{C}(I-\lambda \nabla f) y_{n}\right\| \text {. }
\end{aligned}
$$

From (2.2), we have

$$
\frac{z_{n}-x_{n}}{\theta_{n}}=\frac{\beta_{n}}{\theta_{n}}\left(Q y_{n}-x_{n}\right)+\frac{1-\beta_{n}}{\theta_{n}}\left(T \tilde{y}_{n}-x_{n}\right) .
$$

This together with $\lim _{n \rightarrow \infty} \frac{\left\|z_{n}-x_{n}\right\|}{\theta_{n}}=0$ and $\frac{\beta_{n}}{\theta_{n}}=0$ implies that

$$
\lim _{n \rightarrow \infty} \frac{\left\|T \tilde{y}_{n}-x_{n}\right\|}{\theta_{n}}=0
$$

Hence,

$$
\lim _{n \rightarrow \infty} \frac{\theta_{n}\left\|T \tilde{y}_{n}-x_{n}\right\|}{\beta_{n}}=\lim _{n \rightarrow \infty} \frac{\left\|T \tilde{y}_{n}-x_{n}\right\|}{\theta_{n}} \frac{\theta_{n}^{2}}{\beta_{n}}=0 .
$$

Observe that

$$
y_{n}-x_{n}=\theta_{n}\left(S x_{n}-x_{n}\right) .
$$

Therefore, we get

$$
\lim _{n \rightarrow \infty} \frac{\theta_{n}}{\beta_{n}}\left\|y_{n}-x_{n}\right\|=\lim _{n \rightarrow \infty} \frac{\theta_{n}^{2}}{\beta_{n}}\left\|S x_{n}-x_{n}\right\|=0,
$$


and hence

$$
\begin{aligned}
\frac{\theta_{n}}{\beta_{n}} & \left\|y_{n}-T P_{C}(I-\lambda \nabla f) y_{n}\right\| \\
\leq & \frac{\theta_{n}}{\beta_{n}}\left(\left\|y_{n}-x_{n}\right\|+\left\|x_{n}-T P_{C}(I-\lambda \nabla f) y_{n}\right\|\right) \\
\leq & \frac{\theta_{n}}{\beta_{n}}\left(\left\|y_{n}-x_{n}\right\|+\left\|x_{n}-T P_{C}\left(I-\lambda \nabla f_{\alpha_{n}}\right) y_{n}\right\|\right. \\
& \left.+\left\|T P_{C}\left(I-\lambda \nabla f_{\alpha_{n}}\right) y_{n}-T P_{C}(I-\lambda \nabla f) y_{n}\right\|\right) \\
\leq & \frac{\theta_{n}}{\beta_{n}}\left(\left\|y_{n}-x_{n}\right\|+\left\|x_{n}-T \tilde{y}_{n}\right\|+\left\|\left(I-\lambda \nabla f_{\alpha_{n}}\right) y_{n}-(I-\lambda \nabla f) y_{n}\right\|\right) \\
= & \frac{\theta_{n}}{\beta_{n}}\left\|y_{n}-x_{n}\right\|+\frac{\theta_{n}}{\beta_{n}}\left\|x_{n}-T \tilde{y}_{n}\right\|+\frac{\theta_{n}}{\beta_{n}} \lambda \alpha_{n}\left\|y_{n}\right\| \\
= & \frac{\theta_{n}}{\beta_{n}}\left\|y_{n}-x_{n}\right\|+\frac{\theta_{n}}{\beta_{n}}\left\|x_{n}-T \tilde{y}_{n}\right\|+\frac{\theta_{n}^{2}}{\beta_{n}} \frac{\alpha_{n}}{\theta_{n}} \lambda\left\|y_{n}\right\| \rightarrow 0 \quad \text { as } n \rightarrow \infty .
\end{aligned}
$$

Thus, it follows that

$$
\limsup _{n \rightarrow \infty} \frac{\theta_{n}}{\beta_{n}}\left\langle S x^{*}-x^{*}, y_{n}-x^{*}\right\rangle \leq 0
$$

and hence

$$
\limsup _{n \rightarrow \infty} \frac{2\left(1-(1-\rho) \beta_{n}\right)}{1-\rho} \frac{\theta_{n}}{\beta_{n}}\left\langle S x^{*}-x^{*}, y_{n}-x^{*}\right\rangle \leq 0 .
$$

Utilizing Lemma 2.3, from $\sum_{n=0}^{\infty} \widetilde{M} \alpha_{n}<\infty$ and (3.18)-(3.20), we conclude that the sequence $\left\{x_{n}\right\}$ converges strongly to $x^{*}$. Taking into consideration that $\left\|x_{n}-y_{n}\right\| \rightarrow 0$ and $\left\|x_{n}-z_{n}\right\| \rightarrow 0$, we obtain that $\left\|y_{n}-x^{*}\right\| \rightarrow 0$ and $\left\|z_{n}-x^{*}\right\| \rightarrow 0$ as $n \rightarrow \infty$. This completes the proof.

Remark 3.3 The following parametric sequences satisfy the hypotheses of Theorem 3.2.

(a) $\alpha_{n}=\frac{1}{n^{1+s+t}}, \beta_{n}=\frac{1}{n^{s}}$ and $\theta_{n}=\frac{1}{n^{t}}$, where $t \in\left(0, \frac{1}{3}\right]$ and $s \in(t, 2 t)$ or $t \in\left(\frac{1}{3}, \frac{1}{2}\right)$, $s \in(t, 1-t)$

(b) $\sigma_{n}=\frac{1}{2}+\frac{2}{n}, \gamma_{n}=\delta_{n}=\frac{1}{4}-\frac{1}{n}, \forall n>4$.

Remark 3.4 Theorems 3.1 and 3.2 improve, extend, supplement and develop [11, Theorems 3.1 and 3.2] and [1, Theorems 5.2 and 6.1] in the following aspects:

(a) Three-step iterative algorithm (2.2) with regularization for MP (1.1), two nonexpansive mappings and a strictly pseudocontractive mapping are more flexible and more subtle than the algorithms in $[1,11]$.

(b) The argument techniques in Theorems 3.1 and 3.2 are different from the ones in [11, Theorems 3.1 and 3.2] and the ones in [1, Theorems 5.2 and 6.1] because we use the properties of strict pseudocontractive mappings and maximal monotone mappings (see, for example, Lemmas 2.1, 2.4 and 2.6).

(c) Compared with the proof of Theorems 5.2 and 6.1 in [1], the proof of Theorems 3.1 and 3.2 shows $\lim _{n \rightarrow \infty}\left\|y_{n}-P_{C}\left(I-\lambda \nabla f_{\alpha_{n}}\right) y_{n}\right\|=\lim _{n \rightarrow \infty}\left\|z_{n}-P_{C}\left(I-\lambda \nabla f_{\alpha_{n}}\right) z_{n}\right\|=0$ 
via the argument of $\lim _{n \rightarrow \infty}\left\|\nabla f_{\alpha_{n}}\left(y_{n}\right)-\nabla f(p)\right\|=\lim _{n \rightarrow \infty}\left\|\nabla f_{\alpha_{n}}\left(z_{n}\right)-\nabla f(p)\right\|=0$, $\forall p \in \operatorname{Fix}(T) \cap \operatorname{Fix}(\Gamma) \cap \Xi$ (see Step 3 in the proof of Theorem 3.1).

(e) Theorems 3.1 and 3.2 remove the condition $\operatorname{Fix}(T) \cap$ int $C \neq \varnothing$ in [11, Theorems 3.1 and 3.2].

\section{Competing interests}

The authors declare that they have no competing interests.

\section{Authors' contributions}

All authors read and approved the final manuscript.

\section{Author details}

'Department of Mathematics, Shanghai Normal University, Shanghai 200234, China. ${ }^{2}$ Scientific Computing Key Laboratory of Shanghai Universities, Shanghai, 200234, China. ${ }^{3}$ Department of Mathematics and Statistics, King Fahd University of Petroleum and Minerals, Dhahran, Saudi Arabia. ${ }^{4}$ Department of Mathematics, Aligarh Muslim University, Aligarh, India.

\section{Acknowledgements}

In this research, second and third author were supported by King Fahd University of Petroleum \& Minerals project number IN101009. The first author was partially supported by the National Science Foundation of China (11071169), Innovation Program of Shanghai Municipal Education Commission (09ZZ133) and Leading Academic Discipline Project of Shanghai Normal University (DZL707). The research part of third author was done during his visit to King Fahd University of Petroleum \& Minerals, Dhahran, Saudi Arabia.

Received: 9 July 2013 Accepted: 30 September 2013 Published: 08 Nov 2013

\section{References}

1. Xu, HK: Averaged mappings and the gradient-projection algorithm. J. Optim. Theory Appl. 150, 360-378 (2011)

2. Baillon, JB, Haddad, G: Quelques proprietes des operateurs angle-bornes et $n$-cycliquement monotones. Isr. J. Math. 26, 137-150 (1977)

3. Ceng, LC, Ansari, QH, Yao, JC: Extragradient-projection method for solving constrained convex minimization problems. Numer. Algebra Control Optim. 1, 341-359 (2011)

4. Ceng, LC, Ansari, QH, Wen, CF: Implicit relaxed and hybrid methods with regularization for minimization problems and asymptotically strict pseudocontractive mappings in the intermediate sense. Abstr. Appl. Anal. 2013, Article ID 854297 (2013)

5. Ceng, LC, Ansari, QH, Wen, CF: Multi-step implicit iterative methods with regularization for minimization problems and fixed point problems. J. Inequal. Appl. 2013, Article ID 240 (2013)

6. Ansari, QH, Lalitha, CS, Mehta, M: Generalized Convexity, Nonsmooth Variational Inequalities and Nonsmooth Optimization. CRC Press, Boca Raton (2013)

7. Kinderlehrer, D, Stampacchia, G: An Introduction to Variational Inequalities and Their Applications. Academic Press, New York (1980)

8. Ceng, LC, Ansari, QH, Wong, NC, Yao, JC: Implicit iterative methods for hierarchical variational inequalities. J. Appl. Math. 2012, Article ID 472935 (2012)

9. Cianciaruso, F, Colao, V, Muglia, L, Xu, HK: On implicit methods for variational inequalities via hierarchical fixed point approach. Bull. Aust. Math. Soc. 80, 117-124 (2009)

10. Yao, Y, Liou, YC, Kang, SM: Approach to common elements of variational inequality problems and fixed point problems via a relaxed extragradient method. Comput. Math. Appl. 59, 3472-3480 (2010)

11. Yao, Y, Liou, YC, Marino, G: Two-step iterative algorithms for hierarchical fixed point problems and variational inequality problems. J. Appl. Math. Comput. 31, 433-445 (2009)

12. Byrne, C: A unified treatment of some iterative algorithms in signal processing and image reconstruction. Inverse Probl. 20, 103-120 (2004)

13. Combettes, PL: Solving monotone inclusions via compositions of nonexpansive averaged operators. Optimization 53, 475-504 (2004)

14. Marino, G, Xu, HK: Weak and strong convergence theorems for strict pseudo-contractions in Hilbert spaces. J. Math. Anal. Appl. 329, 336-346 (2007)

15. Xu, HK: Iterative algorithms for nonlinear operators. J. Lond. Math. Soc. 66, 240-256 (2002)

16. Reineermann, J: Uber fixpunkte kontrahierender abbildungen und schwach konvergente Toeplitz-verfahren. Arch. Math. 20, 59-64 (1969)

17. Rockafellar, RT: On the maximality of sums of nonlinear monotone operators. Trans. Am. Math. Soc. 149, 75-88 (1970)

18. Moudafi, A, Mainge, PE: Towards viscosity approximations of hierarchical fixed points problems. Fixed Point Theory Appl. 2006, Article ID 95453 (2006)

19. Moudafi, A, Mainge, PE: Strong convergence of an iterative method for hierarchical fixed point problems. Pac. J. Optim. 3, 529-538 (2007)

10.1186/1687-1812-2013-284

Cite this article as: Ceng et al.: Iterative algorithms with regularization for hierarchical variational inequality

problems and convex minimization problems. Fixed Point Theory and Applications 2013, 2013:284 